\title{
Taylorian points of an algebraic curve and bivariate Hermite interpolation
}

\author{
Len Bos And JeAn-Paul Calvi
}

\begin{abstract}
We introduce and study the notion of Taylorian points of algebraic curves in $\mathbb{C}^{2}$, which enables us to define intrinsic Taylor interpolation polynomials on curves. These polynomials in turn lead to the construction of a wellbehaved Hermitian scheme on curves, of which we give several examples. We show that such Hermitian schemes can be collected to obtain Hermitian bivariate polynomial interpolation schemes.
\end{abstract}

Mathematics Subject Classification (2000): 41A05 (primary); 41A63, 46A32, 14Q05 (secondary).

\section{Introduction}

In classical univariate Lagrange interpolation theory, we know the values of a function at finitely many points and we construct the polynomial of smallest degree which takes the same value at these points. But we may know more than the mere values of the function, we may know its local behavior around the points, that is, its Taylor polynomial at each of the points, each one to a certain order. When we collect these pieces of (local) information and look for the polynomial of smallest degree that matches the same local behavior we find exactly the classical Hermite interpolation polynomial. When working with multivariate functions, apart from the (fundamental) fact that we must now choose the location of the points more carefully, we can follow the same processes. However, knowing the Taylor polynomial, say of degree $d$, of a function of $n$ complex variables $f$ requires the knowledge of all the partial derivatives of order $\leq d$, or, equivalently, the total Fréchet derivatives $f^{(j)}, j=0, \ldots, d$, of $f$ which is a symmetric $j$-linear form on $\left(\mathbb{C}^{n}\right)^{j}$. In many cases, it seems more realistic to know $f^{(j)}$ on a subspace of $\left(\mathbb{C}^{n}\right)^{j}$, for example on the product of $j$ copies of a hyperplane in $\mathbb{C}^{n}$, which amounts to knowing the local behavior of the restriction of the function to that hyperplane. Likewise, in the bivariate Hermite interpolation theory that we introduce and study in this paper, 
the information which is at our disposal is not the local behavior of the whole function but rather the local behavior of its restrictions to (irreducible) algebraic curves. There is no obvious definition of how such local behavior should be measured. We use the local Taylor polynomials on curves that we defined in [4]. The construction is recalled below. The point is that the computation of theses polynomials requires the use of a parameterization of the curve in a neighborhood of the point we consider and, in general, depends on the parameterization we choose. The first part of this paper studies for what conditions (on the point) our local Taylor polynomial are parameterization-free. This leads to our definition of Taylorian points on curves. We show for example that all but finitely many points have such a property. As often occurs in mathematics, what is required for the elegance of a theory turns out to be useful for its applications. Thus, in a second part, our notion of Taylorian points permits us to define well-behaved Hermitian schemes on curves - of which we give various examples - which are collectable, in a sense to made precise, in $\mathbb{C}^{2}$ to construct bivariate Hermitian interpolation schemes. Although the principle is similar, the higher dimensional case $(n>2)$ requires a somewhat different and more technical treatment that we shall present in another paper.

We work exclusively with functions of complex variables. However, with simple adaptations, everything remains true in the real variable case. The passage to the real case is explained in detail in [4].

We use standard notation. The letter $\mathbb{P}$ is reserved to spaces of polynomials. In particular, $\mathbb{P}\left(\mathbb{C}^{n}\right)$ denotes the space of polynomials in $n$ complex variables and $\mathbb{P}^{d}\left(\mathbb{C}^{n}\right)$ the subspace of polynomials of (total) degree at most $d$.

Let us conclude this introduction by pointing out a few other works in multivariate polynomial interpolation which has been a very active field of research in the last decades. The survey paper of Gasca and Sauer [10] gives an account of the many roads along which it evolved, including the ideal theoretic approach initiated by Möller in [17] and subsequently developed as for instance in [16]. The works of Lorenz $[14,15]$ is more particularly dedicated to Hermite interpolation. Recent interesting results around Bojanov-Xu schemes include $[1,11,12]$. The remarkable work of de Boor and Ron $[7,8]$ deserves particular attention. We learn from them the concept of least space which stands at the basis of our own work. Finally, we mention another fruitful but radically different approach (the interpolation conditions are no longer discrete) associated with the names of Kergin and Hakopian which gives rise to various interesting mean-value interpolations see e.g. [2,9] and [5] for a recent contribution.

ACKnowledgements. The research for this paper has been done, in part, when Len Bos was visiting professor at the University Paul Sabatier of Toulouse in May 2007. Several results have been conjectured with the help of the computer algebra systems MAPLE and MAXIMA. 


\section{Taylor interpolation on an irreducible curve}

\subsection{Irreducible algebraic curves}

We denote by $V$ an irreducible algebraic curve in $\mathbb{C}^{2}$ and write $V(q)$ when we want to specify an irreducible polynomial $q$ (which is unique up to scalar multiplication) that defines $V$, that is, $V=\{q=0\}$. The degree of $V(q)$ is the degree of any defining polynomial $q$. We denote by $\mathbb{P}(V)$ the ring of polynomial functions on $V$ and by $\mathbb{P}^{d}(V)$ the subspace of polynomial functions on $V$ of degree at most $d$,

$$
\mathbb{P}^{d}(V)=\left\{p_{\mid V}: p \in \mathbb{P}^{d}\left(\mathbb{C}^{2}\right)\right\} .
$$

An application of the simplest form of the Nullstellensatz (which only requires $q$ to be square free) shows that the kernel of the linear map

$$
\mathbb{P}^{d}\left(\mathbb{C}^{2}\right) \ni p \rightarrow p_{\mid V} \in \mathbb{P}^{d}(V)
$$

is given by $\mathbb{P}^{d}\left(\mathbb{C}^{2}\right) \cap q \cdot \mathbb{P}\left(\mathbb{C}^{2}\right)$. It follows that if the degree of $V$ is equal to $r$, the dimension $N_{d}(V)$ of $\mathbb{P}^{d}(V)$ is given by

$$
N_{d}(V)=\left(\begin{array}{c}
d+2 \\
2
\end{array}\right)-\left(\begin{array}{c}
d-r+2 \\
2
\end{array}\right)
$$

with the convention that $\left(\begin{array}{c}d-r+2 \\ 2\end{array}\right)=0$ when $d<r$. The function $d \rightarrow N_{d}(V)$ is the Hilbert function of the principal ideal generated by $q$.

In Table 1.1, we collect the values of $N_{d}(V)$ that we shall repeatedly use in the sequel.

Table 1.1. The values of the Hilbert function $d \rightarrow N_{d}(V)$ as a function of $\operatorname{deg} q$.

\begin{tabular}{ccl}
$\operatorname{deg} q$ & $N_{d}(V)$ & \\
\hline 1 & $d+1$ & \\
2 & $2 d+1$ & \\
3 & $3 d$ & $(d \geq 1)$ \\
4 & $4 d-2$ & $(d \geq 2)$ \\
$r$ & $r d+\left(3 r-r^{2}\right) / 2$ & $(d \geq r-2)$
\end{tabular}

The complex analytic curve defined by the regular (smooth) points of $V$ - the points $a$ in $V$ for which $D q(a) \neq 0-$ is denoted by $V^{0}$. The irreducibility of $q$ implies that $V^{0}$ is connected. This property is essential in the construction of the local Taylor interpolants (see below). A (local) parameterization of $V$ (and of $V^{0}$ ) at $a \in V^{0}$ is a 3-tuple $\mathcal{L}=(b, \Omega, R)$ where $b \in \mathbb{C}, \Omega$ is an open connected neighborhood of $b$ in $\mathbb{C}$ and $R=\left(R_{1}, R_{2}\right): \Omega \rightarrow \mathbb{C}^{2}$ an analytic function such that $R(b)=a, R(\Omega) \subset V^{0}$ and $R$ is an homeomorphism from $\Omega$ to $R(\Omega)$ (in particular $\left.\left(R_{1}^{\prime}(b), R_{2}^{\prime}(b)\right) \neq(0,0)\right)$. The complex number $b$ is called the base 
point of the parameterization $\mathcal{L}$. Two parameterizations at $a$ differ locally by a complex diffeomorphism. Precisely, if $(c, D, S)$ is another parameterization of $V$ at $a$ then there exist an open neighborhood $\mathcal{U}$ of $c$ in $D$ and a diffeomorphism $h: \mathcal{U} \rightarrow h(\mathcal{U}) \subset \Omega$ such that $S=R \circ h$ on $\mathcal{U}$. Most concrete local parameterizations are obtained via a use of the implicit function theorem. When $V(q)$ is a graph, that is, $q(x, y)=y-s(x)$ with $s \in \mathbb{P}(\mathbb{C})$, then $V^{0}=V$ and, for every $b \in \mathbb{C}$, the parameterization $(b, \mathbb{C}, z \rightarrow(z, s(z)))$ is called the trivial parameterization of $V$ at $(b, s(b))$.

\subsection{Local differential operators}

Let $\mathcal{L}=(b, \Omega, R)$ be a parametrization of $V$ at $a \in V^{0}$. We denote by $\mathbb{P}_{\mathcal{L}}^{d}$ the space of functions on $\Omega$ induced by the polynomials of degree at most $d$ on $V$,

$$
\mathbb{P}_{\mathcal{L}}^{d}:=\mathbb{P}^{d}\left(\mathbb{C}^{2}\right) \circ R=\mathbb{P}^{d}(V) \circ R .
$$

A monomial $(z-b)^{k}$ is called a least b-monomial for $\mathbb{P}_{\mathcal{L}}^{d}$ if there exists $f \in \mathbb{P}_{\mathcal{L}}^{d}$ such that, in a neighborhood of $b, f(z)=(z-b)^{k}+o\left((z-b)^{k}\right)$, equivalently if there exists $p \in \mathbb{P}^{d}\left(\mathbb{C}^{2}\right)$ such that,

$$
(p \circ R)(z)=(z-b)^{k}+(\text { terms of } b \text {-order } \geq k+1) .
$$

By the expression 'terms of $b$-order $\geq k+1$ ' - that we shall often abbreviate to 'terms of higher $b$-order' - we mean an analytic function of the form $\sum_{j=k+1}^{\infty} c_{j}(x-b)^{j}$ in a neighborhood of $b$.

We define the set of integers $\operatorname{pow}(\mathcal{L}, d)$ as

$$
\operatorname{pow}(\mathcal{L}, d)=\left\{k \in \mathbb{N}:(z-b)^{k} \text { is a least } b \text {-monomial for } \mathbb{P}_{\mathcal{L}}^{d}\right\} .
$$

The subspace of $\mathbb{P}(\mathbb{C})$ spanned by the least $b$-monomials is called the least space of $\mathbb{P}_{\mathcal{L}}^{d}$ and is denoted by $\mathbb{P}_{\mathcal{L} \downarrow}^{d}$,

$$
\begin{aligned}
\mathbb{P}_{\mathcal{L} \downarrow}^{d} & =\operatorname{span}\left\{\text { least } b \text {-monomials for } \mathbb{P}_{\mathcal{L}}^{d}\right\} \\
& =\operatorname{span}\left\{(z-b)^{k}: k \in \operatorname{pow}(\mathcal{L}, d)\right\} .
\end{aligned}
$$

By selecting $p=1$ in (2.4)), we see that the least space always contains $1=$ $(z-b)^{0}$, i.e. $0 \in \operatorname{pow}(\mathcal{L}, d)$. The definition also immediately implies

$$
\operatorname{pow}(\mathcal{L}, d) \subset \operatorname{pow}(\mathcal{L}, d+1), \quad d \geq 1 .
$$

It is known [7] that least spaces have the same dimension as their original space. Specifically,, see [4] for the third equality,

$$
\sharp \operatorname{pow}(\mathcal{L}, d)=\operatorname{dim} \mathbb{P}_{\mathcal{L} \downarrow}^{d}=\operatorname{dim} \mathbb{P}_{\mathcal{L}}^{d}=\operatorname{dim} \mathbb{P}^{d}(V) .
$$

In particular, the inclusion in (2.8) is always strict. 
A polynomial $Q$ in $\mathbb{P}_{\mathcal{L} \downarrow}^{d}$ induces a (local) differential operator $\bar{Q}_{\mathcal{L}}(D)$ defined on the space $\mathrm{A}(a)$ of analytic functions on a neighborhood of $a$ in $\mathbb{C}^{2}$ by

$$
\bar{Q}_{\mathcal{L}}(D)(f):=\bar{Q}(D)(f \circ R)
$$

where the right hand term is

$$
\sum_{j} \bar{a}_{j}(f \circ R)^{(j)}(b), \quad \text { if } Q(z)=\sum_{j} a_{j}(z-b)^{j} .
$$

In particular, taking $Q(z)=(z-b)^{k}, k \in \operatorname{pow}(\mathcal{L}, d)$, we obtain the operator $D_{\mathcal{L}}^{k}$ defined by

$$
D_{\mathcal{L}}^{k}(f)=(f \circ R)^{(k)}(b) .
$$

When $k=0$, the corresponding operator is the Dirac functional $f \rightarrow f(a)$. Several examples of such differential operators are given in [4]. Let us just briefly recall the simplest case of a line. If $V=\{-\beta x+\alpha y=0\}$, using the parameterization $\mathcal{L}=\left(t_{0}, \mathbb{C}, z \rightarrow z \cdot(\alpha, \beta)\right)$ at $a=\left(t_{0} \alpha, t_{0} \beta\right)$, the differential operators $D_{\mathcal{L}}^{k}$ are the usual directional derivatives in the direction of the spanning vector $(\alpha, \beta)$ of $V$,

$$
D_{\mathcal{L}}^{k}(f)=\left.\frac{d^{k}}{d z^{k}} f(z \cdot(\alpha, \beta))\right|_{z=t_{0}} .
$$

It is natural to consider (2.12) as it clearly reflects the (local) behavior of $f$ on the curve $V$. However, it is important to note that the operators $D_{\mathcal{L}}^{k}(f)$ are linear combinations of partial derivatives of $f$ at $a$ and of order $\leq k$. The computation of these operators only requires knowing the first $k$ derivatives of $R$ which, in the usual case, is a simple computational problem. We shall turn to this question later.

As follows from the definition $(2.10), \bar{Q}_{\mathcal{L}}(D)$ is well defined on $\mathbb{P}(V)$. Indeed, two polynomials $p_{1}$ and $p_{2}$ which coincide on $V$ only differ by a multiple of $q$ hence, since $q \circ R=0, p_{1} \circ R=p_{2} \circ R$. This is used in Theorem 2.1 below.

Details and more general results on these differential operators can be found in [4].

\subsection{Local Taylor interpolation}

The following theorem is proved in [4]. The proof uses the principle of uniqueness of analytic continuation and this is the reason why we need the connectedness of $V^{0}$, that is, the irreducibility of $V$.

Theorem 2.1. Let $\mathcal{L}$ be a parameterization of the irreducible curve $V$ at $a \in V^{0}$. For every $f \in \mathrm{A}(a)$, there exists a unique polynomial $p \in \mathbb{P}^{d}(V), d \geq 1$, such that

$$
\bar{Q}_{\mathcal{L}}(D)(f)=\bar{Q}_{\mathcal{L}}(D)(p), \quad Q \in \mathbb{P}_{\mathcal{L} \downarrow}^{d} .
$$

This polynomial $p$ is called the $\mathcal{L}$-Taylor polynomial of $f$ and is denoted by $\mathbf{T}_{\mathcal{L}}^{d}(f)$. 
In particular, the linear map $\mathbf{T}_{\mathcal{L}}^{d}: f \in \mathrm{A}(a) \rightarrow \mathbf{T}_{\mathcal{L}}^{d}(f)$ satisfies

$$
\mathbf{T}_{\mathcal{L}}^{d}(p)=p_{\mid V}, \quad p \in \mathbb{P}^{d}\left(\mathbb{C}^{2}\right) .
$$

To check whether a given polynomial $p_{\mid V} \in \mathbb{P}^{d}(V)$ is equal to $\mathbf{T}_{\mathcal{L}}^{d}(f)$ it suffices to verify that

$$
D_{\mathcal{L}}^{k}(f)=D_{\mathcal{L}}^{k}(p), \quad k \in \operatorname{pow}(\mathcal{L}, d) ;
$$

this is because every $\bar{Q}_{\mathcal{L}}(D)$ is a linear combination of the $D_{\mathcal{L}}^{k}$ 's.

Since $\mathbf{T}_{\mathcal{L}}^{d}(f)$ depends only of the restriction of $f$ to $V$, we may speak of $\mathbf{T}_{\mathcal{L}}^{d}(p)$ for $p \in \mathbb{P}(V)$. It is then clear that the content of Theorem 2.1 is purely algebraic. It may be rephrased as follows.

Given complex numbers $\Delta_{k}, k \in \operatorname{pow}(\mathcal{L}, d)$, there exists a unique $P \in \mathbb{P}^{d}(V)$ such that $D_{\mathcal{L}}^{k}(P)=\Delta_{k}, k \in \operatorname{pow}(\mathcal{L}, d)$.

As is emphasized in the notation, the construction of the projector $\mathbf{T}_{\mathcal{L}}^{d}$ depends on the particular parameterization $\mathcal{L}$ we use. We shall now see that, in many cases, the projector is actually independent of the parameterization.

\section{Parameterization-free interpolation}

\subsection{Changing parameterizations}

Let $\mathcal{L}^{1}=\left(b^{1}, \Omega^{1}, R^{1}\right)$ and $\mathcal{L}^{2}=\left(b^{2}, \Omega^{2}, R^{2}\right)$ be two parameterizations of $V^{0}$ at $a$ and $h$ be a local diffeomorphism such that $R^{2}=R^{1} \circ h$ on a neighborhood of $b^{2}$. If $\left(z-b^{1}\right)^{k} \in \mathbb{P}_{\mathcal{L}^{1} \downarrow}^{d}$ then there exists $p \in \mathbb{P}^{d}\left(\mathbb{C}^{2}\right)$ such that

$$
\begin{aligned}
\left(p \circ R^{1}\right)(z)=\left(z-b^{1}\right)^{k}+o\left(\left(z-b^{1}\right)^{k}\right) & \\
\Longrightarrow & \left(p \circ R^{2}\right)(z)=\left(h(z)-b^{1}\right)^{k}+o\left(\left(h(z)-b^{1}\right)^{k}\right) .
\end{aligned}
$$

Since $h(z)-b^{1}=h(z)-h\left(b^{2}\right)=\lambda\left(z-b^{2}\right)+o\left(\left(z-b^{2}\right)\right)$ with $\lambda:=h^{\prime}\left(b^{2}\right) \neq 0$ it follows that

$$
\left(p \circ R^{2}\right)(z)=\lambda^{k}\left(z-b^{2}\right)^{k}+o\left(\left(z-b^{2}\right)^{k}\right) .
$$

Hence the monomial $\left(z-b^{2}\right)^{k}$ belongs to $\mathbb{P}_{\mathcal{L}^{2} \downarrow}$. Thus, different parameterizations produce monomials of the same degree. In particular, we have proved the following:

Lemma 3.1. Two parameterizations $\mathcal{L}^{1}$ and $\mathcal{L}^{2}$ of $V$ at $a \in V^{0}$ with the same base point $b$ have the same least spaces, $\mathbb{P}_{\mathcal{L}^{1} \downarrow}^{d}=\mathbb{P}_{\mathcal{L}^{2} \downarrow}^{d}, \quad d \geq 1$.

In any case, the set $\operatorname{pow}(\mathcal{L}, d)$ does not depend on $\mathcal{L}$ but only on $a$. From now on, we shall denote it by $\operatorname{pow}(a, d)$. 


\subsection{The kernel of $\mathbf{T}_{\mathcal{L}}^{d}$}

The crucial property of $a \in V^{0}$ will be of producing a set pow $(a, d)$ which is gap free. Since $0 \in \operatorname{pow}(a, d)$ and, see (2.9), $\sharp \operatorname{pow}(a, d)=N_{d}(V)$, this means that

$$
\operatorname{pow}(a, d)=\left\{0,1, \ldots, N_{d}(V)-1\right\},
$$

which is equivalent to

$$
\mathbb{P}_{\mathcal{L} \downarrow}^{d}=\operatorname{span}\left\{(x-b)^{k}: k=0, \ldots, N_{d}(V)-1\right\}=\mathbb{P}^{N_{d}(V)-1}(\mathbb{C}) .
$$

As will be apparent from the proofs below, the reason why this property is so important is that, when it holds, we may make use of the Leibniz formula and show that, given a function $g$, the operator $f \rightarrow D_{\mathcal{L}}^{k}(f g)$ is still an operator of the form $\bar{Q}_{\mathcal{L}}(D)$ with $Q \in \mathbb{P}_{\mathcal{L} \downarrow}^{d}$.

Our first theorem relates this property to the nature of the kernel of $\mathbf{T}_{\mathcal{L}}^{d}$.

Theorem 3.2. Let $d \geq 1$. Let $V=\{q=0\} \subset \mathbb{C}^{2}$ be an irreducible algebraic curve, $a=\left(a_{1}, a_{2}\right)$ a regular point of $V$, and $\mathcal{L}=(b, W, R)$ a local parameterization of $V$ at $a$.

(A) If $\mathbf{p o w}(a, d)$ is gap-free then $\operatorname{ker} \mathbf{T}_{\mathcal{L}}^{d}$ is an ideal (of $\mathrm{A}(a)$ ).

(B) Conversely, if $\operatorname{ker} \mathbf{T}_{\mathcal{L}}^{d}$ is an ideal then $\mathbf{p o w}(d, a)$ is gap-free.

Thus, when $\operatorname{ker} \mathbf{T}_{\mathcal{L}}^{d}$, is an ideal for one parameterization then it is an ideal for every parameterization.

Proof. (A) Let $f \in \operatorname{ker} \mathbf{T}_{\mathcal{L}}^{d}$ and $g \in \mathrm{A}(a)$. We must prove that $f g \in \operatorname{ker} \mathbf{T}_{\mathcal{L}}^{d}$ or, equivalently, that the relations $D^{k}(f \circ R)(b)=0$ for $0 \leq k \leq N_{d}(V)-1$ imply $D^{k}(f g \circ R)(b)=0$ for the same $k$. This is an immediate consequence of the ordinary Leibniz formula for

$$
D_{\mathcal{L}}^{k}(f g)=(f g \circ R)^{(k)}(b)=\sum_{j=0}^{k}\left(\begin{array}{l}
k \\
j
\end{array}\right)(f \circ R)^{(j)}(b)(g \circ R)^{(k-j)}(b)=0 .
$$

Indeed, since pow $(a, d)$ is gap-free and $\mathbf{T}_{\mathcal{L}}^{d}(f)=0$, all of the $(f \circ R)^{(j)}(b)$ 's in (3.5) vanish.

(B) We show that if pow $(a, d)$ is not gap-free then $\operatorname{ker} \mathbf{T}_{\mathcal{L}}^{d}$ fails to be an ideal. We define $s$ to be the largest integer $s$ such that $s \notin \operatorname{pow}(a, d)$ but $s+1 \in \operatorname{pow}(a, d)$. We write $R(z)=\left(R_{1}(z), R_{2}(z)\right)$. Recall that at least one of the two numbers $\mu_{1}:=R_{1}^{\prime}(b)$ and $\mu_{2}:=R_{1}^{\prime}(b)$ is different from 0 . We assume that $\mu_{1} \neq 0$. The case $\mu_{2} \neq 0$ is similar. Consider the polynomial $p_{0}(x, y)=\left(x-a_{1}\right)^{s}$. Since, $R_{1}(z)-a_{1}=\mu_{1}(z-b)+o((z-b))$, we have

$$
\begin{aligned}
\left(p_{0} \circ R\right)(z) & =\left(R_{1}(z)-a_{1}\right)^{s} \\
& =\mu_{1}^{s}(z-b)^{s}+\sum_{i=1}^{l} \theta_{i}^{0}(z-b)^{s+i}+(\text { terms of higher } b \text {-order })
\end{aligned}
$$


where $s+i \in \operatorname{pow}(a, d)$ for $i=1, \ldots, l$ and $s+l$ is the greatest element of $\operatorname{pow}(a, d)$. Since $(z-b)^{s+1} \in \mathbb{P}_{\mathcal{L} \downarrow}^{d}$ there exists $q_{1}(x, y)$ such that

$$
\left(q_{1} \circ R\right)(z)=\theta_{1}^{0}(z-b)^{s+1}+(\text { terms of higher } b \text {-order }) .
$$

Setting $p_{1}(x, y)=p_{0}(x, y)-q_{1}(x, y)$, we have

$$
\left(p_{1} \circ R\right)(z)=\mu_{1}^{s}(z-b)^{s}+\sum_{i=2}^{l} \theta_{i}^{1}(z-b)^{s+i}+(\text { terms of higher } b \text {-order })
$$

Continuing in this way, by eliminating successively the coefficients of $(z-b)^{s+i}$, $i=2,3, \ldots, l$, we construct a polynomial $p_{l}(x, y)$ (obviously an element of $\mathrm{A}(a)$ ) such that

$$
\begin{aligned}
\left(p_{l} \circ R\right) & =\mu_{1}^{s}(z-b)^{s}+(\text { terms of } b \text {-order not smaller than } s+l+1) \\
& =\mu_{1}^{s}(z-b)^{s}+E(z) \quad \text { with } E(z)=o\left((z-b)^{s+l}\right) .
\end{aligned}
$$

Now, we claim that $p_{l} \in \operatorname{ker} \mathbf{T}_{\mathcal{L}^{t}}^{d}$. Indeed $\mathbf{T}_{\mathcal{L}}^{d}\left(p_{l}\right)=0$ follows from the fact that for every $t \in \operatorname{pow}(a, d)$ we have $\tilde{D}_{\mathcal{L}}^{t}\left(p_{l}\right)=0$ and this since,

$$
D_{\mathcal{L}}^{t}\left(p_{l}\right)=\left.\frac{d^{t}}{d z^{t}}(z-b)^{s}\right|_{z=b}+\left.\frac{d^{t}}{d z^{t}} E(z)\right|_{z=b}
$$

with $\left.\frac{d^{t}}{d z^{t}}(z-b)^{s}\right|_{z=b}=0$ (because $s \notin \operatorname{pow}(a, d) \Longrightarrow s \neq t$ ), and $\left.\frac{d^{t}}{d z^{t}} E\right|_{z=b}=0$ since $\left.\frac{d^{t}}{d z^{l}}(z-b)^{r}\right|_{z=b}=0$, when $r>s+l(\geq t)$.

On the other hand, the polynomial $P(x, y)=\left(x-a_{1}\right) p_{l}(x, y)$ satisfies

$$
\begin{aligned}
(P \circ R)(z) & =\left(R_{1}(z)-a_{1}\right)\left(\mu_{1}^{s}(z-b)^{s}+(\text { terms of } b \text {-order } \geq s+l+1)\right) \\
& =\mu_{1}^{s+1}(z-b)^{s+1}+(\text { terms of } b \text {-order } \geq s+2) .
\end{aligned}
$$

Hence $(P \circ R)^{(s+1)}(b)=(s+1) ! \mu_{1}^{s+1} \neq 0$ which implies that $D_{\mathcal{L}}^{s+1}(P) \neq 0$ and hence $\mathbf{T}_{\mathcal{L}}^{d}(P) \neq 0$. We have therefore shown that $P \notin \operatorname{ker} \mathbf{T}_{\mathcal{L}}^{d}$ although it is the product of $\left(x-a_{1}\right)$ by an element of $\operatorname{ker} \mathbf{T}_{\mathcal{L}}^{d}$ which is therefore not an ideal.

Remark 3.3. It follows from the proof that the integer $s$ cannot be smaller than $d+1$. Otherwise the polynomial $p_{l}$ would define a nonzero element of $\mathbb{P}^{d}(V)$ and $\mathbf{T}_{\mathcal{L}}^{d}\left(p_{l}\right)=0$ would contradict the fact that $\mathbf{T}_{\mathcal{L}}^{d}$ is a polynomial projector on $\mathbb{P}^{d}(V)$. 


\subsection{Taylorian points}

Let $a$ be a regular point of the irreducible algebraic curve $V$ and let $d \geq 1$. When every parameterization $\mathcal{L}$ of $V$ at $a$ gives the same projector $\mathbf{T}_{\mathcal{L}}^{d}$, this projector is simply denoted by $\mathbf{T}_{a}^{d}$. We then say that the (parameterization-free) Taylor projector at $a$ is well defined and that $a$ is a $d$-Taylorian point (for $V$ ). When $\mathbf{T}_{a}^{d}$ is well defined for every $d \geq 1$, we say that $a$ is $\infty$-Taylorian.

Note that, as in the case of $\mathbf{T}_{\mathcal{L}}^{d}$, when $\mathbf{T}_{a}^{d}$ is well defined, it is also well defined on $\mathbb{P}(V)$.

Theorem 3.4. Let $V$ be an irreducible algebraic curve and $a \in V^{0}$. Then $a$ is $d$-Taylorian $(d \geq 1))$ if and only if $\operatorname{pow}(a, d)$ is gap-free, that is $\operatorname{pow}(a, d)=$ $\left\{0,1, \ldots, N_{d}(V)-1\right\}$.

Proof. We first prove that the condition is sufficient. Let $\mathcal{L}^{1}=\left(b^{1}, \Omega^{1}, R^{1}\right)$ and $\mathcal{L}^{2}=\left(b^{2}, \Omega^{2}, R^{2}\right)$ be two parameterizations of $V$ at $a$. We show that for every $f \in$ $\mathrm{A}(a)$ we have $\mathbf{T}_{\mathcal{L}^{1}}^{d}(f)=\mathbf{T}_{\mathcal{L}^{2}}^{d}(f)$. To simplify the notation, we write $P_{1}=\mathbf{T}_{\mathcal{L}^{1}}^{d}(f)$. Let $\eta$ be a complex diffeomorphism defined on a open neighborhood $\mathcal{O}^{2}$ in $\Omega^{2}$ such that $R^{2}=R^{1} \circ \eta$ on $\mathcal{O}^{2}$. Now, for $k \in \operatorname{pow}(a, d) \backslash\{0\}$, that is $k=1, \ldots, N_{d}(V)-1$, we have

$$
\begin{aligned}
D_{\mathcal{L}^{2}}^{k}\left(P_{1}\right) & =\left(P_{1} \circ R^{2}\right)^{(k)}\left(b^{2}\right) \\
& =\left(P_{1} \circ R^{1} \circ \eta\right)^{(k)}\left(b^{2}\right) \\
& =\sum_{j=1}^{k} C_{j}\left(P_{1} \circ R^{1}\right)^{(j)}\left(b_{1}\right),
\end{aligned}
$$

where the $C_{j}$ 's depend only on the derivatives of $\eta$ at $b^{2}$. Equality (3.13) comes from (3.12) by repeated applications of the chain rule (or, for those who know it, from a direct use of the Faa di Bruno formula). Now, since $P_{1}=\mathbf{T}_{\mathcal{L}^{1}}^{d}(f)$, we have

$$
\begin{aligned}
\left(P_{1} \circ R^{1}\right)^{(j)}\left(b_{1}\right) & =D_{\mathcal{L}^{1}}^{j}\left(P_{1}\right)=D_{\mathcal{L}^{1}}^{j}(f) \\
& =\left(f \circ R^{1}\right)^{(j)}\left(b_{1}\right), \quad j=1, \ldots, N_{d}(V)-1 .
\end{aligned}
$$

From (3.13) and (3.14) we deduce that, for $k \in\left\{1, \ldots, N_{d}(V)-1\right\}$,

$$
\begin{aligned}
D_{\mathcal{L}^{2}}^{k}\left(P_{1}\right) & =\sum_{j=1}^{k} C_{j}\left(f \circ R^{1}\right)^{(j)}\left(b_{1}\right) \\
& =\left(f \circ R^{2}\right)^{(k)}\left(b^{2}\right) \\
& =D_{\mathcal{L}^{2}}^{k}(f) .
\end{aligned}
$$

As for $k=0$,

$$
D_{\mathcal{L}^{2}}^{(0)}\left(P_{1}\right)=P_{1}(a)=f(a)=D_{\mathcal{L}^{2}}^{(0)}(f)
$$


Therefore we must have $P_{1}=\mathbf{T}_{\mathcal{L}^{2}}^{d}(f)$, as was to be proved.

To prove that the condition is necessary, we show that if $\operatorname{pow}(a, d)$ is not gapfree then we can find two parameterizations $\mathcal{L}^{1}$ and $\mathcal{L}^{2}$ and a function $f \in \mathrm{A}(a)$ such that $\mathbf{T}_{\mathcal{L}^{1}}^{d}(f) \neq \mathbf{T}_{\mathcal{L}^{2}}^{d}(f)$.

Let $\mathcal{L}^{1}=\left(b^{1}, \Omega^{1}, R^{1}\right)$ be any parameterization of $V$ at $a$. We use the same construction (and notation) as in the proof of Theorem 3.2 (B). In particular, we use the same integer $s$ and the same polynomial $p_{l}$ satisfying (3.10) for which we have $\mathbf{T}_{\mathcal{L}^{1}}^{d}\left(p_{l}\right)=0$. Now take

$$
\eta(z)=b^{1}+\alpha\left(z-b_{1}\right)+\beta\left(z-b^{1}\right)^{2}
$$

where $\alpha$ and $\beta$ are both nonzero complex numbers and define a new parametrization $\mathcal{L}^{2}=\left(b^{1}, \Omega^{2}, R^{2}\right)$ where $\Omega^{2}$ is a sufficiently small disc centered at $b^{1}$ (so that $\left.\eta\left(\Omega^{2}\right) \subset \Omega^{1}\right)$ and $R^{2}=R^{1} \circ \eta$. Since $\alpha \neq 0, \eta$ is a local diffeomorphism and $\mathcal{L}^{2}$ is therefore well defined. From (3.10), we have

$$
\begin{aligned}
\left(p_{l} \circ R^{2}\right)(z) & =\left(p_{l} \circ R^{1}\right)(\eta(z)) \\
& =\mu_{1}^{s}\left(\eta(z)-b^{1}\right)^{s}+o\left(\left(\eta(z)-b^{1}\right)^{s+l}\right) \\
& =\mu_{1}^{s} \alpha^{s}\left(z-b^{1}\right)^{s}+(s-1) \alpha^{s-1} \beta\left(z-b^{1}\right)^{s+1}+o\left(\left(z-b^{1}\right)^{s+1}\right) .
\end{aligned}
$$

In the last equality, we used the fact that $s+l+1>s+1$. Now the operator $D_{\mathcal{L}^{2}}^{s+1}$ is used in the definition of $\mathbf{T}_{\mathcal{L}^{2}}^{d}$ but, in view of (3.21),

$$
D_{\mathcal{L}^{2}}^{s+1}\left(p_{l}\right)=\left(p_{l} \circ R^{2}\right)^{(s+1)}\left(b^{1}\right)=(s+1) ! \cdot(s-1) \cdot \alpha^{s-1} \cdot \beta .
$$

Hence, since $s \neq 1$ (see remark 3.3) and both $\alpha$ and $\beta$ are different from zero, $D_{\mathcal{L}^{2}}^{s+1}\left(p_{l}\right) \neq 0$. This makes it impossible that $\mathbf{T}_{\mathcal{L}^{2}}\left(p_{l}\right)=0$ and hence $\mathbf{T}_{\mathcal{L}^{1}}^{d}\left(p_{l}\right) \neq$ $\mathbf{T}_{\mathcal{L}^{2}}^{\mathcal{L}^{2}}\left(p_{l}\right)$.

Remark 3.5. To say that $\mathbf{T}_{\mathcal{L}}^{d}$ does not depend on $\mathcal{L}$ does not mean, of course, that the differential operators $D_{\mathcal{L}}^{k}$ or more generally the operators $\bar{Q}_{\mathcal{L}}(D)$ do not depend on $\mathcal{L}$. It only means that their linear span depend only on $a$ and $d$ and not on $\mathcal{L}$.

We omit the proof of the following corollary which is again a simple consequence of the Leibniz formula.

Corollary 3.6. Let $f, g \in \mathrm{A}(a)$. If $\mathbf{T}_{a}^{d}$ is well-defined then

$$
\mathbf{T}_{a}^{d}(f g)=\mathbf{T}_{a}^{d}(f) \mathbf{T}_{a}^{d}(g) \quad \bmod \operatorname{ker} \mathbf{T}_{a}^{d} .
$$

Equivalently,

$$
\mathbf{T}_{a}^{d}(f g)=\mathbf{T}_{a}^{d}\left(\mathbf{T}_{a}^{d}(f) \mathbf{T}_{a}^{d}(g)\right) .
$$

Here, on the right hand side of (3.24) we used the extension of $\mathbf{T}_{a}^{d}$ to $\mathbb{P}(V)$.

The following corollary will play a fundamental role in the construction of our bivariate interpolants in Section 6. 
Corollary 3.7. Let $f, g \in \mathrm{A}(a)$. We assume that $\mathbf{T}_{a}^{d}$ is well-defined. If $f(a) \neq 0$ and $\mathbf{T}_{a}^{d}(f g)=0$ then $\mathbf{T}_{a}^{d}(g)=0$.

Proof. In view of (3.24), we have $\mathbf{T}_{a}^{d}\left(\mathbf{T}_{a}^{d}(f) \mathbf{T}_{a}^{d}(g)\right)=0$ with $\mathbf{T}_{a}^{d}(f) \neq 0$ (for $\left.\mathbf{T}_{a}^{d}(f)(a)=f(a) \neq 0\right)$. An application of [4, Lemma 5] yields $\mathbf{T}_{a}^{d}(g)=0$. Let us sketch a direct proof. Choosing any parameterization $\mathcal{L}=(b, \Omega, R)$ of $V$ at $a$, to show that $\mathbf{T}_{a}^{d}(g)=0$, it suffices to check that $D_{\mathcal{L}}^{k}(g)=0$ for $k \in \operatorname{pow}(a, d)=$ $\left\{0,1, \ldots, N_{d}(V)-1\right\}$. This is easily seen by induction on $k$. First, from $\mathbf{T}_{a}^{d}(f g)=$ 0 , we have $(f g)(a)=0$ and, since $f(a) \neq 0$, we have $0=g(a)=D_{\mathcal{L}}^{0}(g)$. Now, we go from $k$ to $k+1$ by observing that, for some $\lambda_{j}$ 's,

$$
D_{\mathcal{L}}^{k+1}(f g)=f(a) D_{\mathcal{L}}^{k+1}(g)+\sum_{j=0}^{k} \lambda_{j} D_{\mathcal{L}}^{j}(g) .
$$

Hence we get $D_{\mathcal{L}}^{k+1}(g)=0$ from $D_{\mathcal{L}}^{k+1}(f g)=0, f(a) \neq 0$ and the induction hypothesis.

The following example shows that the property is not true in general when $a$ is not $d$-Taylorian, that is, when $\mathbf{T}_{a}^{d}$ is replaced by $\mathbf{T}_{\mathcal{L}}^{d}$.

Example 3.8. Let $V=\left\{y=x^{3}\right\}$. We use the trivial parameterization $\mathcal{L}$ of $V$ at $0=(0,0)$. Since $\mathbb{P}_{\mathcal{L}}^{1}=\operatorname{span}\left\{1, x, x^{3}\right\}$, we have $\operatorname{pow}(0,1)=\{0,1,3\}$ and 0 is not 1 -Taylorian. Define $f(x, y)=1-x$ and $g(x, y)=y+x^{2}$. We have $f(0)=1 \neq 0$ and $\mathbf{T}_{\mathcal{L}}^{1}(f g)=0$. Indeed to say that $D_{\mathcal{L}}^{k}(f g)=0, k=0,1,3$, means that the coefficients of $x^{k}, k=0,1,3$ in $f\left(x, x^{3}\right) \cdot g\left(x, x^{3}\right)$ vanish. This is true since $f\left(x, x^{3}\right) \cdot g\left(x, x^{3}\right)=x^{2}-x^{4}$. On the other hand, it is not true that $\mathbf{T}_{\mathcal{L}}^{1}(g)=0$ since $g\left(x, x^{3}\right)=x^{3}+x^{2}$.

\section{The set of $d$-Taylorian points}

We give a few properties and the main algebraic criterion to decide whether a given point is $d$-Taylorian.

\subsection{Changes of coordinates}

Changes of coordinates systems have no effect on the property of being $d$-Taylorian. Here is a precise statement of this basic but useful property.

Proposition 4.1. Let $V=V(q)$ be an irreducible algebraic curve and let $A$ be an affine isomorphism of $\mathbb{C}^{2}$. A point $a \in V^{0}(q)$ is d-Taylorian for $V(q)$ if and only if $A(a)$ is d-Taylorian for $V\left(q \circ A^{-1}\right)$. 
Proof. If $\mathcal{L}=(b, \Omega, R)$ is a parameterization of $V(q)$ at $a$ then $\mathcal{L}^{A}:=(b, \Omega, A$ 。 $R)$ is a parameterization of $V\left(q \circ A^{-1}\right)$ at $A(a)$. Since $\mathbb{P}^{d}\left(\mathbb{C}^{2}\right)$ is invariant under $A$, we have $\mathbb{P}_{\mathcal{L}}^{d}=\mathbb{P}_{\mathcal{L}^{A}}^{d}$. Hence the least spaces are identical and the conclusion follows.

The property of being $d$-Taylorian really depends on $d$ and not solely on $a$. The following example shows that a point may be $d$-Taylorian without being $d+1$ Taylorian. Another example will be given later showing that a point which is not $d$-Taylorian may be $d^{\prime}$-Taylorian for some $d^{\prime}>d$.

Example 4.2. Let $V=\left\{y=x^{2}+x^{6}\right\}$ and $a=0=(0,0)$. Then $a$ is 1-Taylorian but not 2-Taylorian.

Proof. We use the trivial parameterization $\left(R(z)=\left(z, z^{2}+z^{6}\right)\right)$. Since $N_{1}(V)=3$ and $\mathbb{P}_{\mathcal{L}}^{1}=\left\{1, z, z^{2}+z^{6}\right\}$, we have $\mathbb{P}_{\mathcal{L} \downarrow}^{1}=\operatorname{span}\left\{1, z, z^{2}\right\}=\mathbb{P}^{3-1}(\mathbb{C})$ hence 0 is 1-Taylorian. On the other hand, since

$$
\mathbb{P}^{2}(V)=\operatorname{span}\left\{1, x, y, x^{2}, x y, y^{2}\right\}
$$

we have

$$
\begin{aligned}
\mathbb{P}_{\mathcal{L}}^{2}=\left\{1, z, z^{2}+z^{6}, z^{2}, z\left(z^{2}+z^{6}\right)\right. & \left.,\left(z^{2}+z^{6}\right)^{2}\right\} \\
& \Longrightarrow \mathbb{P}_{\mathcal{L} \downarrow}^{2} \supset \operatorname{span}\left\{1, z, z^{2}, z^{3}, z^{4}, z^{6}\right\}
\end{aligned}
$$

where the monomial $z^{6}$ is seen to be a least monomial on taking the combination $\left(z^{2}+z^{6}\right)-z^{2}$. Since the dimensions of both sides are equal, the inclusion must an equality. There is therefore a gap in $\operatorname{pow}(0,2)(5$ is missing $)$ and 0 is not 2Taylorian.

\subsection{Some examples}

It is readily seen that if every point of a line in $\mathbb{C}^{2}$ is $\infty$-Taylorian. The same is true for a curve of degree 2 .

Proposition 4.3. Every point of an irreducible quadric in $\mathbb{C}^{2}$ is $\infty$-Taylorian.

Proof. This can be found in [4, Section 3.4.4].

As shown by the next result, there is a simple geometric characterization of 1-Taylorian points.

Proposition 4.4. A regular point of an irreducible algebraic curve of degree $q \geq 2$ is 1-Taylorian if and only if it is not an inflection point. In particular, at most $3 q(q-2)$ points are not 1-Taylorian. 
Proof. Let $a=\left(a_{1}, a_{2}\right), \in V^{0}$. We assume without loss of generality that $\partial_{2} q(a) \neq$ 0 and use the parameterization given by the implicit function theorem, $R(z)=$ $(z, r(z))$. We have

$$
\mathbb{P}_{\mathcal{L}}^{1}=\operatorname{span}\{1, z, r(z)\}=\operatorname{span}\left\{1, z-a_{1}, r(z)-r\left(a_{1}\right)\right\} .
$$

Since $r(z)-r\left(a_{1}\right)=r^{\prime}\left(a_{1}\right)\left(z-a_{1}\right)+\frac{1}{2} r^{\prime \prime}\left(a_{1}\right)\left(z-a_{1}\right)^{2}+\cdots$, we have

$$
\mathbb{P}_{\mathcal{L} \downarrow}^{1}=\operatorname{span}\left\{1, z-a_{1},\left(z-a_{1}\right)^{n}\right\}
$$

where $n$ is the smallest integer $>1$ such that $r^{(n)}\left(a_{1}\right) \neq 0$. It follows that $\mathbb{P}_{\mathcal{L} \downarrow}^{1}=$ $\mathbb{P}^{2}(\mathbb{C})$ if and only if $n=2$, that is $r^{\prime \prime}\left(a_{1}\right) \neq$ which occurs if and only if $a$ is not an inflection point of $V$. The classical estimation on the number of inflection points may be found in [13, Proposition 3.33, page 73].

\subsection{The Wronskian criterion}

We now give the basic computational criterion to decide whether, given $V$ and $d \geq 1$, a point $a \in V^{0}$ is $d$-Taylorian for $V$.

Theorem 4.5. Let $V$ be an irreducible algebraic curve. Let $d \geq 1$ and $\left(B_{i}: i=\right.$ $\left.0, \cdots, N_{d}(V)-1\right)$ a basis of $\mathbb{P}^{d}(V)$. Let $a \in V^{0}$ and let $\overline{\mathcal{L}}=(b, \Omega, R)$ be a parameterization of $V$ at $a$. Then $a$ is d-Taylorian if and only if

$$
\operatorname{det} W(V, a) \neq 0
$$

where $W(V, a)$ is the $N_{d}(V) \times N_{d}(V)$ matrix defined by

$$
W_{i j}(V, a)=\left(B_{i} \circ R\right)^{(j)}(b), \quad i, j=0, \ldots, N_{d}(V)-1 .
$$

The matrix $W(V, a)$ depends on $V, d, a, R$ (and $\mathcal{L}))$ and the basis of $\mathbb{P}^{d}(V)$ we choose. However, as follows from Theorem 3.4, the condition (4.2) is independent of $R$. We may write $W(V, a, d)$ if we need to emphasize the dependence on $d$. Note that $W(V, a, d)$ is a sub-matrix of $W(V, a, d+1)$ provided that we work with a basis of $\mathbb{P}^{d+1}(V)$ which extends that of $\mathbb{P}^{d}(V)$.

Proof. To say that $a$ is $d$-Taylorian means that the following linear problem has a solution

$$
\begin{aligned}
& \forall j=0, \ldots, N_{d}(V)-1 \exists C_{i}^{[j]}, i=0, \ldots, N_{d}(V)-1, \quad \text { such that } \\
& \sum_{i=0}^{N_{d}(V)-1} C_{i}^{[j]}\left(B_{i} \circ R\right)(z)=(z-b)^{j}+o\left((z-b)^{j}\right) .
\end{aligned}
$$


If this problem has a solution so does

$$
\begin{array}{r}
\forall j=0, \ldots, N_{d}(V)-1 \exists C_{i}^{[j]}, i=0, \ldots, N_{d}(V)-1, \quad \text { such that } \\
\sum_{i=0}^{N_{d}(V)-1} C_{i}^{[j]}\left(B_{i} \circ R\right)(z)=(z-b)^{j}+o\left((z-b)^{N_{d}(V)-1}\right) .
\end{array}
$$

Indeed having (all) the solutions of (4.4), we obtain (all) the solutions of (4.5) by using the same elimination process as we did in the proof of Theorem 3.2 (B). Since the converse is obviously true, both problems are equivalent. But the equation

$$
\sum_{i=0}^{N_{d}(V)-1} C_{i}^{[j]}\left(B_{i} \circ R\right)(z)=(z-b)^{j}+o\left((z-b)^{N_{d}(V)-1}\right)
$$

in turn is equivalent to

$$
\sum_{i=0}^{N_{d}(V)-1} C_{i}^{[j]} \mathbf{T}_{b}^{N_{d}(V)-1}\left(B_{i} \circ R\right)(z)=(z-b)^{j},
$$

where $\mathbf{T}_{b}^{N_{d}(V)-1}$ denotes the ordinary (univariate) Taylor projector at $b$ and to the order $N_{d}(V)-1$. Hence, (4.5) will be solvable if and only the polynomials $\mathbf{T}_{b}^{N_{d}(V)-1}\left(B_{i} \circ R\right), i=0, \ldots, N_{d}(V)-1$, form a basis of $\mathbb{P}^{N_{d}(V)-1}(\mathbb{C})$. Now, it suffices to observe that $W(V, a)$ is the matrix of the coefficients of these polynomials in the basis of $\mathbb{P}^{N_{d}(V)-1}(\mathbb{C})$ formed by the monomials $(z-b)^{j} / j$ !.

Remark 4.6. It is sometimes preferable to normalize $W$ in another manner and choose

$$
W_{i j}(V, a)=\text { coeff. of }(z-b)^{j} \text { in the Taylor expansion of }\left(B_{i} \circ R\right) .
$$

(This amounts to working with $(z-b)^{j}$ in the very last step of the previous proof.)

In view of Theorem 4.5, the problem of deciding whether a given point is $d$ Taylorian requires only to know the first $N_{d}(V)-1$ derivatives of $R$ at the base point $b$. Using the expression (4.8), We actually have

$$
W_{i j}(V, a)=W_{i j}(V, a)=\text { coeff. of }(z-b)^{j} \text { in }\left(B_{i} \circ \mathbf{T}_{b}^{N_{d}(V)-1}(R)\right) .
$$

In practice, say when $\partial_{2} q(a) \neq 0$, the first $k$ values of the derivatives of $R$ at $a_{1}$ are obtained by looking for coefficients $\alpha_{i}, i=1, \ldots, k$ such that

$$
q\left(a_{1}+z, a_{2}+\alpha_{1} z+\ldots \alpha_{k} z^{k}\right)=o\left(z^{k}\right) \quad(z \rightarrow 0) .
$$

The coefficients $\alpha_{i}$ are solutions of a triangular system. Another approach would be to use implicit differentiations of $q$, but this is probably a much less efficient method. 


\subsection{Examples}

Example 4.7. If $V$ is an irreducible curve of degree $\geq 3$ with a symmetry axis through $a \in V^{0}$ then $a$ is not 2-Taylorian.

Proof. In view of Proposition 4.1, we may assume that $a=(0, \tau)$ and the symmetry axis is $x=0$. Since $\operatorname{deg} q \geq 3$, we have $N_{2}(V)=5$ and a (conveniently ordered) basis of $\mathbb{P}^{2}(V)$ is $\left(1, y, y^{2}, x, x y, y^{2}\right)$. By expressing $x$ as a function of $y$, the implicit function theorem gives a parameterization with base point 0 and, because $x=0$ is a symmetry axis, an even function $R: z \rightarrow(r(z), z)$ such that

$$
\mathbf{T}_{0}^{5} r(z)=\tau+\alpha z^{2}+\beta z^{4},
$$

where $\alpha$ and $\beta$ are certain complex numbers. The coefficients of the corresponding matrix $W(V, a)$ in the form (4.8) are given by the following table

\begin{tabular}{c|cccccc} 
& 1 & $y$ & $y^{2}$ & $x$ & $x y$ & $x^{2}$ \\
\hline 1 & 1 & 0 & 0 & $\tau$ & 0 & $\tau^{2}$ \\
$z$ & 0 & 1 & 0 & 0 & $\tau$ & 0 \\
$z^{2}$ & 0 & 0 & 1 & $\alpha$ & 0 & $2 \alpha \tau$. \\
$z^{3}$ & 0 & 0 & 0 & 0 & $\alpha$ & 0 \\
$z^{4}$ & 0 & 0 & 0 & $\beta$ & 0 & $\alpha^{2}$ \\
$z^{5}$ & 0 & 0 & 0 & 0 & $\beta$ & 0
\end{tabular}

It follows that

$$
\operatorname{det} W(V, a)=\left|\begin{array}{ccc}
0 & \alpha & 0 \\
\beta & 0 & \alpha^{2} \\
0 & \beta & 0
\end{array}\right|=0
$$

and $a$ is not 2-Taylorian.

Example 4.8. Let $V=\left\{x^{3}-x^{2}=y^{2}\right\}$. The point $a=(1,0)$ is 3-Taylorian (although, according to the previous example, it is not 2-Taylorian).

Proof. We have $N_{3}(V)=9$. A basis of $\mathbb{P}^{3}(V)$ is

$$
\left(1, y, y^{2}, y^{3}, x, x y, x y^{2}, x^{2}, x^{2} y\right) .
$$

Let $r$ denote the function expressing $x$ as a function of $y$ in a neighbourhood of the origin. We readily find that the Taylor expansion of $r$ at 0 to the order $9-1=8$ is

$$
\mathbf{T}_{0}^{8}(r)(z)=1+z^{2}-2 z^{4}+7 z^{6}-30 z^{8} .
$$

A simplification similar to that of the previous example occurs so that, to compute $\operatorname{det} W(V, a)$, it suffices to know the coefficients of $x, x y, x y^{2}, x^{2}, x^{2} y$ on $z^{4}, z^{5}, z^{6}$, 
$z^{7}, z^{8}$ (with $y=z$ and $\left.x=\mathbf{T}_{0}^{8}(r)(z)\right)$. We find

$$
\operatorname{det} W(V, a)=\left|\begin{array}{rrrrr}
-2 & 0 & 1 & -3 & 0 \\
0 & -2 & 0 & 0 & -3 \\
7 & 0 & -2 & 10 & 0 \\
0 & 7 & 0 & 0 & 10 \\
-30 & 0 & 7 & -42 & 0
\end{array}\right|=-5 \neq 0
$$

and $a$ is 3-Taylorian.

Another method is available here because the curve has a simple useful polynomial parameterization. We use this parameterization in a slightly more general case. We define the following family of elliptic curves,

$$
V_{c}:=\left\{y^{2}=c x^{2}-x^{3}\right\}, \quad c \in \mathbb{C} .
$$

Theses curves are parameterized by the polynomial map $Q(z)=(x(z), y(z))$ with $x(z)=c-z^{2}$ and $y(z)=z\left(c-z^{2}\right)$ ), see [6, Example 8 page 24 and 11 page 26].

Example 4.9. If $c \neq 0$, there are exactly 5 points on $V_{c}$ which are not 2-Taylorian and 9 points which are not 3-Taylorian, two of them not being 2-Taylorian either. If $c=0$, all points but $(0,0)$ are 2-Taylorian and 3-Taylorian.

Proof. We use the parameterization $\mathcal{L}=(b, \mathbb{C}, Q)$ of $V_{c}$ at $a=Q(b)$ with $Q$ as above. Using the expression (4.3), a calculation shows that

$$
\operatorname{det} W\left(V_{c}, a, 2\right)=K b\left(b^{2}+3 c\right)\left(3 b^{2}+c\right)
$$

where $K$ is a constant independent from $c$. Thus when $c \neq 0$, the five points which are not 2-Taylorian are $(c, 0),(4 c, \pm 4 c \sqrt{-3 c}),(2 c / 3, \pm(2 c / 3) \sqrt{-c / 3})$ and when $c=0$, det $W\left(V_{c}, a, 2\right)$ vanishes only for $b=0$, that is $a=(0,0)$. As for the 3-Taylorian points, we have

$$
\operatorname{det} W\left(V_{c}, a, 3\right)=K^{\prime}\left(3 b^{2}+c\right)\left(3 b^{6}+27 c b^{4}+33 c^{2} b^{2}+c^{3}\right)
$$

which have $3 b^{2}+c$ as a common factor with $\operatorname{det} W\left(V_{c}, a, 2\right)$.

\subsection{Most points are $\infty$-Taylorian}

We show that the generic situation for a regular point on an irreducible algebraic curve is to be $\infty$-Taylorian.

Theorem 4.10. Let $V$ be an irreducible algebraic curve and $d \geq 1$. All but finitely many points of $V$ are d-Taylorian. 
Proof. Let $V=V(q)$. Let $\Xi_{2}$ be the set of (regular) points of $V$ such that $\partial q_{2}(a) \neq$ 0 . For $a=\left(a_{1}, a_{2}\right) \in \Xi_{2}$, we use $\mathcal{L}=\left(a_{1}, \Omega, R: z \rightarrow(z, r(z))\right)$, the parameterization given by the implicit function theorem, so that $q(z, r(z))=0$ on $\Omega$. By differentiating the relation $r^{\prime}(z)=-\left(\partial_{1} q / \partial_{2} q\right)(z, r(z))$, it is not difficult to see that the derivatives of $r$ at $a_{1}$ are of the form

$$
r^{(d)}\left(a_{1}\right)=\frac{Q_{d}(a)}{\left(\partial_{2} q(a)\right)^{2 d-1}}
$$

where $Q_{d}$ is a polynomial whose coefficients depends only on $q$, i.e., are independent of $a \in \Xi_{2}$. In fact, a straightforward calculation shows the following recurrence relation:

$$
\begin{aligned}
Q_{d+1}=\left[\partial_{2} q\right]^{2} \cdot\left[\partial_{1} Q_{d}\right]-\left[\partial_{2} q\right] \cdot\left[\partial_{1} q\right] \cdot\left[\partial_{2} Q_{d}\right] \\
\quad+(2 d-1)\left[\partial_{1} q\right] \cdot\left[\partial_{22} q\right] \cdot Q_{d}-(2 d-1)\left[\partial_{2} q\right] \cdot\left[\partial_{12} q\right] \cdot Q_{q} .
\end{aligned}
$$

Now, the entries of the matrix $W(V, a)$ are rational functions of $a$ whose denominators are powers of $\partial_{2} q(a)$, and hence the same can be said of det $W(V, a)$. We let $\Theta_{2}$ denote the numerator of det $W(V, a)$ (choosing, say, as denominator, the smallest possible power of $\partial_{2} q(a)$. Likewise, starting from $\Xi_{1}$ the set of (regular) points such that $\partial q_{1}(a) \neq 0$, we construct a polynomial $\Theta_{1}$. A point $a \in V^{0}$ is not $d$ Taylorian if it lies on $V \cap Z$ where $Z=\left\{\Theta_{1} \Theta_{2}=0\right\}$. Hence, by Bezout's theorem, either the set of points which are not $d$-Taylorian is finite or $q$ (which is irreducible) divides $\Theta_{1} \Theta_{2}$, in which case no point is $d$-Taylorian. It remains to show that the second alternative cannot occur. Let $a$ be any regular point and $(b, \Omega, R)$ be any parameterization of $V^{0}$ at $a$. We observe that for $\alpha \in \Omega,(\alpha, \Omega, R)$ is a parameterization of $V^{0}$ at $R(\alpha)$. This means that the function $\alpha \in \Omega \rightarrow \operatorname{det} W(V, R(\alpha))$, which is the Wronskian of the functions $B_{i} \circ R\left(0 \leq i \leq N_{d}(V)-1\right)$ identically vanishes on $\Omega$. By a well-known property of the Wronskian, this implies that the $B_{i}(R(\alpha))$ 's are linearly dependent on $\Omega$, that is, for some $\lambda_{i}$ 's, not all equal to 0 , $\sum_{i=1}^{N_{d}(V)} \lambda_{i} B_{i}(R(\alpha))=0, \quad \alpha \in \Omega$. Since $V^{0}$ is connected, a use of the principle of unique continuation of analytic functions (see [4]) shows that $\sum_{i=1}^{N_{d}(V)} \lambda_{i} B_{i}=0$ on $V$ and this contradicts the fact that the $B_{i}$ 's form a basis of $\mathbb{P}^{d}(V)$.

\subsection{Taylorian points on graphs of degree 3 and 4}

We have already seen in the proofs of Examples 4.7 and 4.8 that the choice of a convenient basis of $\mathbb{P}^{d}(V)$ may considerably simplify the computation of det $W(a, d)$. A careful examination of this idea in the case of graphs leads to the following results.

Theorem 4.11. Let $V=\{y=s(x)\}$ with $\operatorname{deg} s=3$. The inflection point of $V$ is the unique point which is not $\infty$-Taylorian and it is $d$-Taylorian for no value of $d$. 
Theorem 4.12. Let $V=\{y=s(x)\}$ with $\operatorname{deg} s=4$ and $s$ monic. A point $a=$ $(t, s(t)) \in V$ is $d$-Taylorian, $d \geq 2$, if and only if

$$
F(t)-\frac{1}{40 \cdot 6^{4}}(d+1)(d-2)\left(s^{\prime \prime \prime}(t)\right)^{5} \neq 0
$$

with

$$
F(t)=-\frac{1}{6^{3}} s^{\prime \prime}(t)\left(s^{\prime \prime \prime}(t)\right)^{3}+\frac{1}{2^{3}}\left(s^{\prime \prime}\right)^{2}(t) s^{\prime \prime \prime}(t) .
$$

In view of Proposition 4.1, there is no loss of generality in supposing that $s$ is monic. Recall that the case $d=1$ is treated in Proposition 4.4.

Corollary 4.13. On a graph $V=\left\{y=x^{4}+c_{3} x^{3}+c_{2} x^{2}+c_{1} x+c_{0}\right\}$ of degree 4 , for every $d \geq 2$, there is a maximum of 5 points which are not $d$-Taylorian. The point with abscissa $x=-c_{3} / 4$ is $d$-Taylorian for no value of $d \geq 2$. Any other point may fail to be $d$-Taylorian only for one value of $d, d \geq 2$.

Proof. This follows from the fact that $s^{\prime \prime \prime}(t)$ can be factored out from the polynomial (of degree 5) on the left hand side of (4.18) and when $s^{\prime \prime \prime}(t) \neq 0,(4.18)$ cannot hold for two distinct values of $d$.

The proof we give below, in principle, can be used for a polynomial $s$ of any degree (we shall make this obvious in the proof). But as soon as $\operatorname{deg} s \geq 5$, the condition is complicated and seemingly difficult to use. It should be possible, however, to obtain a precise bound on the maximal number of points which are not $d$-Taylorian on a graph of degree $k, k \in \mathbb{N}^{\star}$.

Proofs of Theorems 4.11 and 4.12. First of all we observe that, again in view of Proposition 4.1, to say that $a=\left(t_{0}, s\left(t_{0}\right)\right)$ is $d$-Taylorian for $\{y=s(x)\}$, is equivalent to saying that $0=(0,0)$ is $d$-Taylorian for $\left\{y+s\left(t_{0}\right)=s\left(t_{0}+x\right)\right\}$, that is, for $\{y=S(x)\}$ where

$$
S(x)=\sum_{j=1}^{\operatorname{deg} s} \frac{s^{(j)}\left(t_{0}\right)}{j !} x^{j} .
$$

Thus, to establish Theorems 4.11 and 4.12, we just need to prove the following two statements.

(A) If $S(x)=c_{1} x+c_{2} x^{2}+x^{3}$ (we may assume that $S$ is monic) then 0 is $d$ Taylorian for $\{y=S(x)\}$ if and only if $c_{2} \neq 0$.

(B) If $S(x)=c_{1} x+c_{2} x^{2}+c_{3} x^{3}+x^{4}$ then 0 is $d$-Taylorian, $d \geq 2$, for $\{y=S(x)\}$ if and only if

$$
F-\frac{6}{40}(d+1)(d-2) c_{3}^{5} \quad \text { with } F=-2 c_{2} c_{3}^{3}+3 c_{2}^{2} c_{3}
$$


We begin by discussing the general case for which $\operatorname{deg} S=k, k \geq 2$ and $d>k$. The standard basis of $\mathbb{P}^{d}(V), V=\{y=S(x)\}$, consists of the monomials $x^{j} y^{l}$ with $l=0, \ldots, d, j=0, \ldots, k-1$ and $i+j \leq d$. In the table below, we arrange the elements of this basis in a sequence $\left(B_{i}\right)$ such that $n_{i}:=\operatorname{deg}\left(B_{i}(x, S(x))\right)$ is an increasing function of $i$.

$$
\begin{array}{cccccccc}
1 & x & x^{2} & \ldots & \ldots & \ldots & x^{k-1} & L_{1} \\
y & x y & x^{2} y & \ldots & \ldots & \ldots & x^{k-1} y & L_{2} \\
\cdots & \ldots & \cdots & \ldots & \ldots & \ldots & \ldots & \\
y^{d-k+1} & x y^{d-k+1} & x^{2} y^{d-k+1} & \ldots & \ldots & \ldots & x^{k-1} y^{d-k+1} & L_{d-k} \\
\hline & & & & & & \\
y^{d-k+2} & x y^{d-k+2} & x^{2} y^{d-k+2} & \ldots & & x^{k-2} y^{d-k+2} & & L_{d-k+1} \\
y^{d-k+3} & x y^{d-k+3} & x^{2} y^{d-k+3} & \ldots & x^{k-3} y^{d-k+3} & & & L_{d-k+2} \\
\ldots & \ldots & & & & & \\
y^{d-2} & x y^{d-2} & x^{2} y^{d-2} & & & & & L_{d-1} \\
y^{d-1} & x y^{d-1} & & & & & & L_{d} \\
y^{d} & & & & & & & L_{d+1}
\end{array}
$$

Thus, for example, we have $B_{0}(x, y)=1, B_{k-1}(x, y)=x^{k-1}, B_{k+1}(x, y)=y$ and $B_{N_{d}(V)-1}(x, y)=y^{d}$. Any missing entry in a line of the above table represents a gap in the sequence $n_{i}$. The first gap occurs at the line $L_{d-k+1}$, between $x^{k-2} y^{d-k+2}$ and $y^{d-k+3}$ for which we go from degree $n=k(d-k+2)+(k-2)$ to degree $k(d-k+3)=n+2$.

Using this basis (and the trivial parameterization of $V=\{y=S(x)\}$ at 0 ) together with the definition (4.8) we obtain a matrix $W(0, d)$ of the form

$$
W(0, d)=\left(\begin{array}{cc}
U & M \\
0 & A
\end{array}\right),
$$

where $U$ is a upper triangular matrix of dimension $\tau=(k-2)+k(d-k+2)+1$ withs ones on the diagonal - its entries are the coefficients of the $B_{i}(x, S(x))$, $i=0, \ldots,(k-2)+k(d-k+2)$ in the basis $1, x, \ldots, x^{\tau}-$ and $A$ is a square matrix of dimension

$$
N_{d}(V)-\tau=\frac{(k-2)(k-1)}{2} .
$$

Since

$$
\operatorname{det} W(0, d)=\operatorname{det} A,
$$

the problem of computing det $W(0, d)$ has been reduced to that of computing the determinant of a matrix whose dimension no longer depends on $d$.

We now turn to the particular cases for which $k=3$ and $k=4$.

(C) When $k=3, N_{d}(V)=3 d$ and, in view of (4.21), $A$ is of dimension 1 and is given by

$$
A=\mathrm{C}\left(x^{3 d-1}, S^{d}(x)\right)
$$


that is, $A$ is the coefficient of $x^{3 d-1}$ in $S^{d}(x)$.

(D) When $k=4, N_{d}(V)=4 d-2, A$ is of dimension 3 and is given by

$$
\begin{array}{cccc}
y^{d-1} & x y^{d-1} & y^{d} \\
x^{4 d-5} & x^{4 d-4} \\
x^{4 d-3}
\end{array}\left(\begin{array}{ccc}
\mathrm{C}\left(x^{4 d-5}, y^{d-1}\right) & \mathrm{C}\left(x^{4 d-5}, x y^{d-1}\right) & \mathrm{C}\left(x^{4 d-5}, y^{d}\right) \\
\mathrm{C}\left(x^{4 d-4}, y^{d-1}\right) & \mathrm{C}\left(x^{4 d-4}, x y^{d-1}\right) & \mathrm{C}\left(x^{4 d-4}, y^{d}\right) \\
\left.\mathbf{C} x^{4 d-3}, y^{d-1}\right) & \mathrm{C}\left(x^{4 d-3}, x y^{d-1}\right) & \mathrm{C}\left(x^{4 d-3}, y^{d}\right)
\end{array}\right),
$$

where $\mathrm{C}\left(x^{i}, x^{j} y^{l}\right)$ denotes the coefficient of $x^{i}$ in $x^{j} S^{l}(x)$.

The computation of the entries of the matrices is a problem of elementary combinatorics.

To compute (4.22), observe that

$$
\mathrm{C}\left(x^{3 d-1},\left(x^{3}+c_{2} x^{2}+c_{1} x\right)^{d}\right)=\mathrm{C}\left(x^{2 d-1},\left(x^{2}+c_{2} x+c_{1}\right)^{d}\right)=d c_{2}
$$

from which follows the assertion (A). The computation of the coefficients of $A$ in (4.23) is somewhat more cumbersome but five of them are easily obtained. From

$$
\begin{aligned}
y^{d-1} & =\left(x^{4}+c_{3} x^{3}+c_{2} x^{2}+c_{1} x\right)^{d-1} \\
& =x^{4 d-4}+(d-1) c_{3} x^{4 d-5}+\cdots,
\end{aligned}
$$

we have

$\mathrm{C}\left(x^{4 d-3}, y^{d-1}\right)=0, \quad \mathrm{C}\left(x^{4 d-4}, y^{d-1}\right)=1 \quad$ and $\quad \mathrm{C}\left(x^{4 d-5}, y^{d-1}\right)=(d-1) c_{3}$.

From the relation $\mathrm{C}\left(x^{i}, x y^{d-1}\right)=\mathrm{C}\left(x^{i-1}, y^{d-1}\right)$, we further get

$$
\mathrm{C}\left(x^{4 d-3}, x y^{d-1}\right)=1 \quad \text { and } \quad \mathrm{C}\left(x^{4 d-4}, x y^{d-1}\right)=(d-1) c_{3} .
$$

Next,

$$
\mathrm{C}\left(x^{4 d-5}, x y^{d-1}\right)=\mathrm{C}\left(x^{4 d-6}, y^{d-1}\right)=\mathrm{C}\left(x^{3 d-5},\left(x^{3}+c_{3} x^{2}+c_{2} x+c_{1}\right)^{d-1}\right) .
$$

To compute this number we must look for partitions $\square=\left(\square_{3}, \square_{2}, \square_{1}, \square_{0}\right)$ such that

$$
\begin{aligned}
3 d-5 & =\square_{3} \cdot 3+\square_{2} \cdot 2+\square_{1} \cdot 1+\square_{0} \cdot 0 \quad \text { and } \\
d-1 & =\square_{3}+\square_{2}+\square_{1}+\square_{0} .
\end{aligned}
$$

Every such partition induces the term

$$
\left(\begin{array}{c}
d-1 \\
\square_{3}, \square_{2}, \square_{1}, \square_{0}
\end{array}\right) c_{3}^{\square_{2}} c_{2}^{\square_{1}} c_{1}^{\square_{0}} \quad \text { in } \quad \mathrm{C}\left(x^{4 d-5}, x y^{d-1}\right) .
$$


The following table gives the two possible partitions and the corresponding terms

$$
\begin{aligned}
3 d-5 & =d-2 \cdot 3+0 \cdot 2+1 \cdot 1+0 \cdot 0 \rightarrow(d-1) c_{2}, \\
& =d-3 \cdot 3+2 \cdot 2+0 \cdot 1+0 \cdot 0 \rightarrow\left(\begin{array}{c}
d-1 \\
2
\end{array}\right) c_{3}^{2} .
\end{aligned}
$$

Thus we have proved

$$
\mathrm{C}\left(x^{4 d-5}, x y^{d-1}\right)=\left(\begin{array}{c}
d-1 \\
2
\end{array}\right) c_{3}^{2}+(d-1) c_{2}
$$

All the other coefficients are computed in a similar way. We give the corresponding arrays without further details.

- $\mathrm{C}\left(x^{4 d-3}, y^{d}\right)=\mathrm{C}\left(x^{3 d-3},\left(x^{3}+c_{3} x^{2}+c_{2} x+c_{1}\right)^{d}\right)$. From

$$
\begin{aligned}
3 d-3 & =d-1 \cdot 3+0 \cdot 2+0 \cdot 1+1 \cdot 0 \rightarrow d c_{1}, \\
& =d-2 \cdot 3+1 \cdot 2+1 \cdot 1+0 \cdot 0 \rightarrow 2\left(\begin{array}{l}
d \\
2
\end{array}\right) c_{2} c_{3}, \\
& =d-3 \cdot 3+3 \cdot 2+0 \cdot 1+0 \cdot 0 \rightarrow\left(\begin{array}{l}
d \\
3
\end{array}\right) c_{3}^{3} .
\end{aligned}
$$

we deduce that

$$
\mathrm{C}\left(x^{4 d-3}, y^{d}\right)=d c_{1}+2\left(\begin{array}{l}
d \\
2
\end{array}\right) c_{2} c_{3}+\left(\begin{array}{l}
d \\
3
\end{array}\right) c_{3}^{3} \text {. }
$$

- $\mathrm{C}\left(x^{4 d-4}, y^{d}\right)=\mathrm{C}\left(x^{3 d-4},\left(x^{3}+c_{3} x^{2}+c_{2} x+c_{1}\right)^{d}\right)$. From

$$
\begin{aligned}
3 d-4 & =d-2 \cdot 3+1 \cdot 2+0 \cdot 1+1 \cdot 0 \rightarrow 2\left(\begin{array}{l}
d \\
2
\end{array}\right) c_{1} c_{3}, \\
& =d-2 \cdot 3+0 \cdot 2+2 \cdot 1+0 \cdot 0 \rightarrow\left(\begin{array}{l}
d \\
2
\end{array}\right) c_{2}^{2}, \\
& =d-3 \cdot 3+2 \cdot 2+1 \cdot 1+0 \cdot 0 \rightarrow 3\left(\begin{array}{l}
d \\
3
\end{array}\right) c_{3}^{2} c_{2}
\end{aligned}
$$

we deduce that

$$
\mathrm{C}\left(x^{4 d-4}, y^{d}\right)=\left(\begin{array}{l}
d \\
2
\end{array}\right) c_{2}^{2}+2\left(\begin{array}{l}
d \\
2
\end{array}\right) c_{1} c_{3}+3\left(\begin{array}{l}
d \\
3
\end{array}\right) c_{3}^{2} c_{2}+\left(\begin{array}{l}
d \\
4
\end{array}\right) c_{3}^{4}
$$


- $\mathrm{C}\left(x^{4 d-5}, y^{d}\right)=\mathrm{C}\left(x^{3 d-5},\left(x^{3}+c_{3} x^{2}+c_{2} x+c_{1}\right)^{d}\right)$. From

$$
\begin{aligned}
3 d-5 & =d-2 \cdot 3+0 \cdot 2+1 \cdot 1+1 \cdot 0 \rightarrow 2\left(\begin{array}{l}
d \\
2
\end{array}\right) c_{1} c_{2}, \\
& =d-3 \cdot 3+2 \cdot 2+0 \cdot 1+1 \cdot 0 \rightarrow 3\left(\begin{array}{l}
d \\
3
\end{array}\right) c_{1} c_{3}^{2}, \\
& =d-3 \cdot 3+1 \cdot 2+2 \cdot 1+0 \cdot 0 \rightarrow 3\left(\begin{array}{l}
d \\
3
\end{array}\right) c_{2}^{2} c_{3}, \\
& =d-4 \cdot 3+3 \cdot 2+1 \cdot 1+0 \cdot 0 \rightarrow 4\left(\begin{array}{l}
d \\
4
\end{array}\right) c_{2} c_{3}^{3}, \\
& =d-5 \cdot 3+5 \cdot 2+0 \cdot 1+0 \cdot 0 \rightarrow\left(\begin{array}{l}
d \\
5
\end{array}\right) c_{3}^{5} .
\end{aligned}
$$

we deduce that

$$
\mathrm{C}\left(x^{4 d-5}, y^{d}\right)=2\left(\begin{array}{l}
d \\
2
\end{array}\right) c_{1} c_{2}+4\left(\begin{array}{l}
d \\
3
\end{array}\right)\left(c_{1} c_{3}^{2}+c_{2}^{2} c_{3}\right)+3\left(\begin{array}{l}
d \\
4
\end{array}\right) c_{2} c_{3}^{3}+\left(\begin{array}{l}
d \\
5
\end{array}\right) c_{3}^{5} .
$$

Hence the matrix $A$ is

$$
\left(\begin{array}{ccc}
(d-1) c_{3}\left(\begin{array}{c}
d-1 \\
2
\end{array}\right) c_{3}^{2}+(d-1) c_{2} & 2\left(\begin{array}{l}
d \\
2
\end{array}\right) c_{1} c_{2}+4\left(\begin{array}{l}
d \\
3
\end{array}\right)\left(c_{1} c_{3}^{2}+c_{2}^{2} c_{3}\right) \\
1 & +3\left(\begin{array}{l}
d \\
4
\end{array}\right) c_{2} c_{3}^{3}+\left(\begin{array}{l}
d \\
5
\end{array}\right) c_{3}^{5} \\
0 & (d-1) c_{3} & \left(\begin{array}{l}
d \\
2
\end{array}\right) c_{2}^{2}+2\left(\begin{array}{l}
d \\
2
\end{array}\right) c_{1} c_{3}+3\left(\begin{array}{l}
d \\
3
\end{array}\right) c_{3}^{2} c_{2}+\left(\begin{array}{l}
d \\
4
\end{array}\right) c_{3}^{4} \\
1 & d c_{1}+2\left(\begin{array}{l}
d \\
2
\end{array}\right) c_{2} c_{3}+\left(\begin{array}{l}
d \\
3
\end{array}\right) c_{3}^{3}
\end{array}\right)
$$

An easily automatized computation yields

$$
\operatorname{det} A=\frac{1}{6} d(d-1)(2 d-1)\left\{\frac{6}{40}(d-2)(d+1) c_{3}^{5}+2 c_{2} c_{3}^{3}-3 c_{2}^{2} c_{3}\right\} .
$$

The assertion (B) follows. The proof we gave works for $d \geq k$. An examination of the remaining cases shows that the statements remains true for $d=1,2$ when $k=3$, and $d=2,3$ when $d=4$.

Corollary 4.14 (to the proof). When $V$ is graph of degree $k$ then for every $a \in V$ and $d>k$,

$$
\operatorname{pow}(a, d) \supset\left\{0,1,2, \ldots, d k-k^{2}+3 k-2\right\},
$$

that is, no gap occurs before $d k-k^{2}+3 k-2$. Moreover, the property that a is a $d$-Taylorian point depends only on the computation of a matrix whose dimension is independent of $d$, namely of dimension $\left(\begin{array}{c}k-1 \\ 2\end{array}\right)$. 


\section{Hermitian schemes on curves}

\subsection{Definition}

Let $V$ be an irreducible algebraic curve in $\mathbb{C}^{2}$ and, for $i=1, \ldots, k$, let $d_{i} \in \mathbb{N}$ and $a_{i}$ be a $d_{i}$-Taylorian point of $V$. We say that

$$
S=\left\{\left(a_{i}, d_{i}\right): i=1, \ldots, k\right\}
$$

is a Hermitian scheme for $\mathbb{P}^{d}(V)$ - we shall also speak of a Hermitian scheme of degree $d$ (for $V$ ) - if, for every function $f$ suitably defined, there exists a unique polynomial $p \in \mathbb{P}^{d}(V)$ such that

$$
\mathbf{T}_{a_{i}}^{d_{i}}(p)=\mathbf{T}_{a_{i}}^{d_{i}}(f), \quad i=1, \ldots, k .
$$

The unique polynomial $p$ in (5.2) will be denoted as $\mathbf{H}_{S}(f)$. The integer $d_{i}$ in (5.1) is called the order of $a_{i}$ in $S$. Here, we may have $d_{i}=0$. In that case, no assumption is made on $a_{i}$ apart from being on $V$ and $T_{a_{i}}^{0}(f)$ simply means $f\left(a_{i}\right)$. In fact, when every $d_{i}$ equals 0 , then $\mathbf{H}_{S}(f)$ is the ordinary Lagrange interpolation polynomial of $f$ on the curve $V$. On the other hand, when $k=1$ then $d_{1}$ must equal $d$ and $\mathbf{H}_{S}(f)=\mathbf{T}_{a_{1}}^{d}(f)$. An immediate necessary condition for $S$ to be a Hermitian scheme of degree $d$ is that

$$
N_{d_{1}}(V)+N_{d_{2}}(V)+\cdots+N_{d_{k}}(V)=N_{d}(V)
$$

This arithmetic condition sets some limitations on the structure of an Hermitian scheme.

Example 5.1. The following table gives the possible structure of a Hermitian scheme of degree 1 and 2 on a curve of degree 4 .

\begin{tabular}{l|l} 
& $S=\left\{\left(a_{1}, d_{1}\right), \ldots,\left(a_{k}, d_{k}\right)\right\}$ \\
\hline \multirow{3}{*}{$d=1$} & $k=1$ (Taylor interpolation), \\
& $k=3$ (Lagrange interpolation) \\
\hline \multirow{5}{*}{$d=2$} & $k=1$ (Taylor interpolation), \\
& $k=2$ (with $\left.\left\{\left(a_{1}, 1\right),\left(a_{2}, 1\right)\right\}\right)$, \\
& $k=4$ (with $\left.\left\{\left(a_{1}, 1\right),\left(a_{2}, 0\right),\left(a_{3}, 0\right),\left(a_{4}, 0\right)\right\}\right)$, \\
& $k=6$ (Lagrange interpolation).
\end{tabular}

If $V$ is a line, any scheme satisfying the arithmetic condition (5.3) - in that case $\sum_{i=1}^{k}\left(d_{i}+1\right)=d+1-$ is a Hermitian scheme. More interesting examples are given below. 
As usual to decide whether a given scheme $H$ is Hermitian, we must show that certain linear functionals are linearly independent, and, when this is the case, $\mathbf{H}_{S}$ is a linear map from $\mathrm{A}\left(\left\{a_{i}, i=1, \ldots, k\right\}\right)$ to $\mathbb{P}^{d}(V)$ which extends to $\mathbb{P}(V)$. The extension is then a projector on $\mathbb{P}^{d}(V)$.

A scheme $S$ satisfying (5.3) is not Hermitian if and only if there exists a nonzero $p \in \mathbb{P}^{d}(V)$ satisfying $\mathbf{T}_{a_{i}}^{d_{i}}(p)=0$ for $i=0, \ldots, k$. Observe also that the property of being a Hermitian scheme is invariant under coordinate changes, that is, if $A$ is a bijective affine mapping of $\mathbb{C}^{2}$ then $S=\left\{\left(a_{i}, d_{i}\right),: i=1, \ldots, k\right\}$ is Hermitian of degree $d$ for $V(q)$ if and only if $A(S)=\left\{\left(A\left(a_{i}\right), d_{i}\right),: i=1, \ldots, k\right\}$ is Hermitian for $\mathbb{P}^{d}\left(V\left(q \circ A^{-1}\right)\right)$.

Lemma 5.2. Let $S=\left\{\left(a_{i}, d_{i}\right): i=1, \ldots, k\right\}$ be a Hermitian scheme of degree $d$ for $V$. Let $f$ and $g$ be analytic on a neighborhood of the points in $S$. If $f\left(a_{i}\right) \neq 0$ for $i=1, \ldots, k$ and $\mathbf{H}_{S}(f g)=0$ then $\mathbf{H}_{S}(g)=0$.

Proof. The assumption $\mathbf{H}_{S}(f g)=0$ simply means $\mathbf{T}_{a_{i}}^{d_{i}}(f g), i=1, \ldots, k$. Since $f\left(a_{i}\right) \neq 0, k$ applications of corollary 3.7 gives $\mathbf{T}_{a_{i}}^{d_{i}}(g)=0$ for every $i$, hence $\mathbf{H}_{S}(g)=0$.

\subsection{The case of a quadric}

Every scheme satisfying the arithmetic condition is Hermitian when $V$ is a quadric.

Proposition 5.3. Let $V$ be an irreducible curve of degree 2, every scheme $S=$ $\left\{\left(a_{i}, d_{i}\right): i=1, \ldots, k\right\}$ is Hermitian provided that

$$
k+2 \sum_{i=1}^{k} d_{i}=2 d+1
$$

Recall that, in view of Proposition 4.3, every point of a quadric is $\infty$-Taylorian.

Proof. By performing a change of variables we may assume that $V=\left\{y=x^{2}\right\}$ or $V=\left\{x^{2}+y^{2}=1\right\}$. We only treat the second case and take $S$ as in the theorem. We consider the classical rational map

$$
R=\left(R_{1}, R_{2}\right): t \in \mathbb{C} \backslash\{ \pm i\} \rightarrow\left(\frac{1-t^{2}}{1+t^{2}}, \frac{2 t}{1+t^{2}}\right)
$$

which is obtained by considering the intersections of $V$ with the lines through $(-1,0)$. We have $R(\mathbb{C} \backslash\{ \pm i\})=V \backslash(-1,0)$ and, if every $a_{i}$ is different from $(-1,0)$, for some $t_{i}$ we have $a_{i}=R\left(t_{i}\right)$ and $\mathcal{L}_{i}=\left(t_{i}, \mathbb{C} \backslash\{ \pm i\}, R\right)$ is a local parameterization of $a_{i}$. In the case where some of the $a_{i}$ 's equals $(-1,0)$, we merely use a similar rational parameterization using line through a point $P$ different from all 
the $a_{i}$ 's. Now, we assume that $S$ is not Hermitian, that is, there exists $p \in \mathbb{P}^{d}\left(\mathbb{C}^{2}\right)$, with $p_{\mid V} \neq 0$, such that

$$
\mathbf{T}_{a_{i}}^{d_{i}}(p)=0, \quad i=1, \ldots, k
$$

This means that

$$
D_{\mathcal{L}_{i}}^{j}(p)=0, \quad j=0, \ldots, 2 d_{i}, \quad i=1, \ldots, k,
$$

which is equivalent to

$$
\left.\frac{d^{j}}{d t^{j}} p\left(\frac{1-t^{2}}{1+t^{2}}, \frac{2 t}{1+t^{2}}\right)\right|_{t=t_{i}}=0, \quad j=0, \ldots, 2 d_{i}, \quad i=0, \ldots, k
$$

Now, there is a polynomial $q \in \mathbb{P}^{2 d}(\mathbb{C})$ such that

$$
p\left(\frac{1-t^{2}}{1+t^{2}}, \frac{2 t}{1+t^{2}}\right)=\frac{q(t)}{\left(1+t^{2}\right)^{d}} .
$$

We claim that (5.5) is equivalent to

$$
\left.\frac{d^{j}}{d t^{j}} q(t)\right|_{t=t_{i}}=0 \quad j=0, \ldots, 2 d_{i}, \quad i=1, \ldots, k
$$

Since $\operatorname{deg} q \leq 2 d$, classical Hermite interpolation theory then implies that $q=0$ contradicting the fact that $p$ is nonzero on $V$. To see that conditions (5.5) and (5.6) are identical, it suffices to observe that $p\left(R\left(t_{i}\right)\right)=0$ if and only if $q\left(t_{i}\right)=0$, then, since

$$
\left.\frac{d}{d t}(P(R(t)))\right|_{t=t_{i}}=\left.\left(1+t^{2}\right)^{-d} \frac{d}{d t} q(t)\right|_{t=t_{i}}
$$

we have

$$
\left.\frac{d}{d t}(P(R(t)))\right|_{t=t_{i}}=0 \text { if and only if }\left.\frac{d}{d t}(q(t))\right|_{t=t_{i}}=0
$$

and so on.

\subsection{Graphs of degree 3}

The above result is no longer true for curves of higher degree. But the property of being Hermitian remains extremely simple in the case of a cubic graph. 
Proposition 5.4. Let $V=\{y=\sigma(x)\}$ with $\sigma(x)=x^{3}+c_{2} x^{2}+c_{1} x+c_{0}, d \geq 1$ and a scheme $S$ in which we separate the points of order zero $\left(d_{i}=0\right)$ and of positive $\operatorname{order}\left(d_{i}>0\right)$,

$$
S=\left\{\left(a_{1}, 0\right), \ldots,\left(a_{j}, 0\right),\left(a_{j+1}, d_{j+1}\right), \ldots,\left(a_{k}, d_{k}\right)\right\},
$$

where $a_{i}=\left(\alpha_{i}, s\left(\alpha_{i}\right)\right)$ is a $d_{i}$-Taylorian point of $V, i=1, \ldots, k$, and

$$
j+3 \sum_{i=j+1}^{d} d_{j}=3 d .
$$

Then $S$ is Hermitian for $\mathbb{P}^{d}(V), d \geq 1$, if and only if

$$
\alpha_{1}+\cdots+\alpha_{j}+3\left(d_{j+1} \alpha_{j+1}+\cdots+d_{k} \alpha_{k}\right)+d c_{2} \neq 0
$$

We arranged the points of $A$ in such a way that $d_{l}=0$ for $1 \leq l \leq j$ and $d_{l}>0$ for $j<l \leq k$. Note that (5.8) is the arithmetic condition (5.3). This condition implies that the number of points of zero order must be a multiple of 3 . The following is an immediate rewriting of the previous proposition.

Proposition 5.5. We make the same assumptions as above and define $P_{S} \in \mathbb{P}^{3 d}(\mathbb{C})$ as

$$
P_{S}(x):=\left(x-\alpha_{0}\right) \cdots\left(x-\alpha_{j}\right)\left(x-\alpha_{j+1}\right)^{3 d_{j+1}} \cdots\left(x-\alpha_{k}\right)^{3 d_{k}} .
$$

Then $S$ is Hermitian if and only if the polynomial $P_{S}-\sigma^{d}$ is exactly of degree $3 d-1$.

To see that Propositions 5.4 and 5.5 are equivalent, it suffices to observe that the left hand side of (5.9) is the (negative of the) coefficient of $x^{3 d-1}$ in $P_{S}-\sigma^{d}$.

Proof. Recall that, given two polynomials $P$ and $Q, Q \neq 0$, we may uniquely expand $P$ in powers of $Q$, that is, if $\operatorname{deg} P=k \operatorname{deg} Q+r$ with $0 \leq r<\operatorname{deg} Q$, there exist unique polynomials $P_{i}$ 's with $\operatorname{deg} p_{(i)}<\operatorname{deg} Q$ such that

$$
P=P_{0} Q^{k}+P_{1} Q^{k-1}+\cdots+P_{k} Q+P_{k+1} .
$$

The polynomials $P_{i}$ are obtained by using repeated Euclidean division by $Q$. We use the expansion (5.11) with $P=P_{S}$ and $Q=\sigma$. Since $\operatorname{deg} P_{S}=\operatorname{deg} \sigma^{d}$ and both polynomials are monic, we have $P_{S, 0}=1$ and the expansion is of the form

$$
P_{S}=\sigma^{d}+P_{S, 1} \sigma^{d-1}+\cdots+P_{S, d} \sigma+P_{S, d+1}, \quad \operatorname{deg} P_{S, i} \leq 2, \quad 1 \leq i \leq d+1 .
$$

Define $q \in \mathbb{P}\left(\mathbb{C}^{2}\right)$ by

$$
q(x, y)=y^{d}+P_{S, 1}(x) y^{d-1}+\cdots+P_{S, d}(x) y+P_{S, d+1}(x) .
$$


The crucial observation is that this polynomial $q$ belongs to $\mathbb{P}^{d}\left(\mathbb{C}^{2}\right)$ if and only if the coefficient of $x^{2}$ on $P_{S, 1}$ equals 0 , that is, if the coefficient of $x^{3 d-1}$ in $P_{S}-\sigma^{d}$ equals 0 . Now, supposing that this is true and using the trivial parameterization $\mathcal{L}_{i}$ at $\alpha_{i}$, we have

$$
\begin{aligned}
D_{\mathcal{L}_{i}}^{j} q & =\left.\frac{d^{j}}{d x^{j}} q(x, \sigma(x))\right|_{x=\alpha_{i}} \\
& =\left.\frac{d^{j}}{d x^{j}} P_{S}(x)\right|_{t=\alpha_{i}}=0, \quad 0 \leq j \leq N_{d_{i}}(V), \quad i=1, \ldots, k .
\end{aligned}
$$

The last inequality follows from the definition of $P_{S}$.

We have therefore find a nonzero polynomial $q_{\mid V}$ such that $\mathbf{T}_{a_{i}}^{d_{i}}(q)=0$ for $i=1, \ldots, k$ and this shows that $S$ is not Hermitian.

Conversely, if $S$ is not Hermitian, we may find a nonzero polynomial $q \in$ $\mathbb{P}^{d}(V)$ such that $D_{\mathcal{L}_{i}}^{j} q$ for $0 \leq j \leq N_{d_{i}}(V)$ and $i=0, \ldots, k$. Using the standard basis of $\mathbb{P}^{d}(V)$ we may write this polynomial $q$ as

$$
q(x, y)=c_{d}^{0} y^{d}+\left(c_{d-1}^{1} x+c_{d-1}^{0}\right) y^{d-1}+\sum_{i=0}^{d-2}\left(c_{i}^{2} x^{2}+c_{i}^{1} x+c_{i}^{0}\right) y^{i} .
$$

That the $D_{\mathcal{L}_{i}}^{j} q$ 's equal 0 means that the univariate polynomial $q(x, \sigma(x))$ vanishes at every $\alpha_{i}$ and to the order $3 d_{i}$ for $i>j$. Hence $P_{S}$ divides $p$ and, since $\operatorname{deg} q(x, \sigma(x)) \leq 3 d$, we must have $q(x, \sigma(x))=K P_{S}(x)$ where $K \neq 0$ since $q_{\mid V} \neq 0$. We may assume that $K=1$. It follows that

$$
P_{S}(x)=c_{d}^{0} \sigma^{d}(x)+\left(c_{d-1}^{1} x+c_{d-1}^{0}\right) \sigma^{d-1}(x) \sum_{i=0}^{d}\left(c_{i}^{2} x^{2}+c_{i}^{1} x+c_{i}^{0}\right) \sigma^{i}(x)
$$

and, because of the uniqueness of the expansion of $P_{S}$ in powers of $\sigma$, the comparison of (5.12) and (5.16) shows that the coefficient of $x^{2}$ in $P_{S, 1}(x)$ which is the coefficient of $x^{3 d-1}$ in $P_{S}-\sigma^{d}$ equals 0 .

As in the classical case, the property of being Hermitian is equivalent to the non-vanishing of a Vandermonde-like determinant. We mention without proof the value of this determinant in the case of pairwise distinct points. A general formula may be obtained using continuity properties.

Proposition 5.6. Let $V=\{y=\sigma(x)\}$ with $\sigma(x)=x^{3}+c_{2} x^{2}+c_{1} x+c_{0}$ and let $a_{i}=\left(\alpha_{i}, \sigma\left(\alpha_{i}\right)\right), i=0, \ldots, 3 d-1$ be $3 d$ pairwise distinct points. If $B=\left(B_{i}, i=\right.$ $0, \ldots, 3 d-1)$ denotes the basis of $\mathbb{P}^{d}(V)$ used in the proof of Theorem 4.11 , that is

$$
B=\left(1, x, x^{2}, y, y x, y x^{2}, \ldots, y^{d-2}, y^{d-2} x, y^{d-2} x^{2}, y^{d-1}, y^{d-1} x, y^{d}\right),
$$

then

$$
\operatorname{det}\left(B_{i}\left(a_{j}\right)\right)=\left\{\sum_{j=0}^{3 d-1} \alpha_{i}+d c_{2}\right\} \cdot \prod_{0 \leq i<j \leq 3 d-1}\left(b_{j}-b_{i}\right)
$$




\subsection{Graphs of degree 4}

In principle, the method we used in the proof of Proposition 5.5 works for a graph of every degree. The condition on $S$, in general, is much more complicated, but the method should enable one to derive qualitative results on the structure of Hermitian schemes on any graph. We hope to turn to this question in a future paper. Here, we shall limit ourselves to give some elements regarding the case of a graph of degree 4 .

Proposition 5.7. Let $V=\{y=\sigma(x)\}$ with $\sigma(x)=x^{4}+c_{3} x^{3}+c_{2} x^{2}+c_{1} x+c_{0}$, $d \geq 2$ and a scheme $S=\left\{\left(a_{1}, d_{1}\right), \ldots,\left(a_{k}, d_{k}\right)\right\}$ where $a_{i}=\left(\alpha_{i}, s\left(\alpha_{i}\right)\right)$ is a $d_{i}$ Taylorian point of $V, i=1, \ldots, k$, and

$$
\sharp\left\{i: d_{i}=0\right\}+3 \cdot \sharp\left\{i: d_{i}=1\right\}+\sum_{d_{i} \geq 2}\left(4 d_{i}-2\right)=4 d-2 .
$$

We associate to $S$ the polynomial $P_{S} \in \mathbb{P}^{4 d-2}(\mathbb{C})$ defined by

$$
P_{S}(x):=\prod_{d_{i}=0}\left(x-\alpha_{i}\right) \cdot \prod_{d_{i}=1}\left(x-\alpha_{i}\right)^{3} \cdot \prod_{d_{i} \geq 2}\left(x-\alpha_{i}\right)^{4 d_{i}-2},
$$

and the polynomial $Q_{S} \in \mathbb{P}^{4 d}(\mathbb{C})$ defined by

$$
Q_{S}(x):=P_{S} \cdot\left(x^{2}-\Delta x-\Gamma\right),
$$

where

$$
\begin{aligned}
& \Delta=\mathrm{C}\left(x^{4 d-3}, P_{S}\right)-\mathrm{C}\left(x^{4 d-1} \sigma^{d}\right) \\
& \Gamma=-\mathrm{C}\left(x^{4 d-2}, \sigma^{d}\right)+\mathrm{C}\left(x^{4 d-4}, P_{S}\right)-\Delta \mathrm{C}\left(x^{4 d-3}, P_{S}\right),
\end{aligned}
$$

where as usual $\mathrm{C}\left(x^{l}, P\right)$ denotes the coefficient of $x^{l}$ in $P$. Then $S$ is Hermitian if and only if the third polynomial $Q_{S, 2}$ in the expansion of $Q_{S}$ in powers of $\sigma$ is exactly of degree 3.

Of course, we have

$$
-\Delta=d c_{3}+\left\{\sum_{d_{i}=0} \alpha_{i}+3 \sum_{d_{i}=1} \alpha_{i}+\sum_{d_{i} \geq 2}\left(4 d_{i}-2\right) \alpha_{i}\right\},
$$

and it is not difficult to also express $\Gamma$ as a function of the roots of $P_{S}$ and of the coefficients of $\sigma$.

Proof (sketch). We have

$$
Q_{S}=\sigma^{d}+Q_{S, 1} \sigma^{d-1}+Q_{S, 2} \sigma^{d-2}+\cdots+Q_{S, d} \sigma+Q_{S, d+1},
$$


where, a priori, $\operatorname{deg} Q_{S, j} \leq 3$. The definition of $\Delta$ and $\Gamma$ gives $\operatorname{deg}\left(Q_{S, 1}\right)=1$. Thus if the assumption on $Q_{S, 2}$ is not true, that is, $\operatorname{deg} Q_{S, 2} \leq 2$, we have

$$
Q_{S}(x)=q(x, \sigma(x))
$$

with $q \in \mathbb{P}^{d}\left(\mathbb{C}^{2}\right)$ (and $q_{\mid V} \neq 0$ ). We then show as in the proof of Proposition 5.5 that

$$
D_{\mathcal{L}_{i}}^{j} q=0, \quad 0 \leq j \leq d_{i}, \quad i=0, \ldots, k,
$$

and this proves that $S$ is not Hermitian.

Conversely, if $S$ is not Hermitian then there exists a nonzero $q \in \mathbb{P}^{d}(V)$ of the form

$$
q(x, y)=\sum_{i=0}^{q} q_{i}(x) y^{i}, \quad \operatorname{deg} q_{i} \leq 3, \operatorname{deg} q_{i}+i \leq d,
$$

such that (5.23) holds true. From the relations

$$
\left.\frac{d^{j}}{d x^{j}} q(x, \sigma(x))\right|_{x=\alpha_{i}}=0, \quad 0 \leq j \leq d_{i}, \quad i=0, \ldots, k,
$$

we deduce that $P_{S}(x)$ divides (the nonzero polynomial) $q(x, \sigma(x))$. Now, observe that the constant $q_{0}$ in (5.24) cannot be equal to 0 because otherwise the degree of $q(x, \sigma(x))$ would be not greater than $4 d-3$ which, together with (5.25) would imply $q(x, \sigma(x))=0$ which is contrary to our assumption. We may therefore assume that $q_{0}=1$. In these conditions, the comparison of the degrees of $P_{S}(x)$ and $q(x, \sigma(x))$ shows that, for some coefficients $\delta$ and $\gamma$,

$$
q(x, \sigma(x))=P_{S}(x) \cdot\left(x^{2}-\delta x-\gamma\right) .
$$

On the other hand, since $\operatorname{deg} q_{1}(x) \leq 1$, we have $\operatorname{deg} q(x, \sigma(x))-\sigma^{d}(x) \leq 4(d-$ $1)+1=4 d-3$. It follows that the coefficients of $x^{4 d-1}$ and $x^{4 d-2}$ in $q(x, \sigma(x))-$ $\sigma^{d}(x)$ must be equal to 0 which is easily seen to imply $\delta=\Delta$ and $\gamma=\Gamma$. We thus have $q(x, \sigma(x))=Q_{S}$ and it only remains to note that the condition ' $\operatorname{deg} q_{2} \leq 2$ ' is now equivalent to the condition ' $Q_{S, 2}$ is not of degree 3 '.

Example 5.8. Let $V=\left\{y=x^{4}\right\}$. We want to study on what conditions the scheme

$$
S=\{(a, 1),(b, 1)\}, \quad a=\left(\alpha, \alpha^{4}\right), b=\left(\beta, \beta^{4}\right), \in V
$$

is Hermitian (for $\mathbb{P}^{2}(V)$ ). The arithmetic condition is plainly satisfied $(3+3=$ $N_{2}(V)$ ) and, in view of Proposition 4.4, to be 1-Taylorian, $a$ and $b$ must simply be taken distinct from 0 . Using the notation of the above proposition, we have

$$
Q_{S}(x)=(x-\alpha)^{3}(x-\beta)^{3}\left(x^{2}-\Delta x-\Gamma\right) .
$$

A calculation shows

$$
\Delta=3 \beta+3 \alpha \text { and } \Gamma=6 \beta^{2}+9 \alpha \beta+6 \alpha^{2} .
$$


Next, since in that case $d-2=0$, the condition that $Q_{S, 2}$ is exactly of degree 3 means that the coefficient of $x^{3}$ in the remainder of the division of $Q_{S}(x)-\sigma^{2}(x)=$ $Q_{S}(x)-x^{8}$ by $x^{4}$ is different from 0 . This condition is easily shown to be

$$
(\beta+\alpha)\left(\beta^{4}+8 \alpha \beta^{3}+10 \alpha^{2} \beta^{2}+8 \alpha^{3} \beta+\alpha^{4}\right) \neq 0 .
$$

Interestingly enough, since

$$
\begin{aligned}
\left(\beta^{4}+\right. & \left.8 \alpha \beta^{3}+10 \alpha^{2} \beta^{2}+8 \alpha^{3} \beta+\alpha^{4}\right) \\
= & \{(2-\sqrt{2}-\sqrt{5-4 \sqrt{2}}) \beta+\alpha\}\{(2-\sqrt{2}+\sqrt{5-4 \sqrt{2}}) \beta+\alpha\} \\
& \times\{(\sqrt{2}+2-\sqrt{4 \sqrt{2}+5}) \beta+\alpha\}\{(\sqrt{4 \sqrt{2}+5}+\sqrt{2}+2) \beta+\alpha\}
\end{aligned}
$$

the (abscissae of the) singular points for the scheme (5.26) are located on five lines intersecting at the origin.

\section{Application: bivariate Hermite interpolation}

\subsection{Collecting schemes}

Let $V_{i}=\left\{q_{i}=0\right\}, i=1, \ldots, m$ be a family of $m$ pairwise distinct irreducible curves in $\mathbb{C}^{2}$ and, for each $i$, let $S_{i}$ be a Hermitian scheme of degree $s_{i}$ on $V_{i}$. We say that the family of schemes $\left\{S_{i}, i=1, \ldots, m\right\}$ is collectable of degree $d$ if for every suitably defined function $f$, there exists a unique polynomial $p \in \mathbb{P}^{d}\left(\mathbb{C}^{2}\right)$ satisfying

$$
\mathbf{H}_{S_{i}}(p)=\mathbf{H}_{S_{i}}(f), \quad i=1, \ldots, m .
$$

As usual, there is an immediate arithmetic condition in order that $\left\{S_{i}, i=1, \ldots, m\right\}$ be collectable. We must have

$$
\sum_{i=1}^{m} N_{s_{i}}\left(V_{i}\right)=\left(\begin{array}{c}
d+2 \\
2
\end{array}\right)
$$

There must therefore be some connection between $d$, the degree $s_{i}$ 's of the schemes $S_{i}$ 's and the degree $r_{i}$ of the curve $V_{i}$. The following theorem gives a simple way of correctly combining these numbers. It contains as particular cases, on one hand, the configurations of points introduced in [3] (for which all the collected scheme are lagrangian) and, on the other hand, a result established in [4, Theorem 4] for which all the collected scheme are Taylorian).

Theorem 6.1. Let $m \geq 2$ and, for $i=1,2, \ldots, m$, let $V_{i}=\left\{q_{i}=0\right\}$ where $q_{i}$ is an irreducible polynomial of degree $r_{i} \geq 1$ in $\mathbb{P}\left(\mathbb{C}^{2}\right)$. Let $d \in \mathbb{N}$ be such that

$$
r_{1}+r_{2}+\cdots+r_{m-1}<d \leq r_{1}+r_{2}+\cdots+r_{m-1}+r_{m} .
$$


We define the integers $s_{i}$ by the relation

$$
\left\{\begin{array}{l}
s_{1}=d \\
s_{i}=d-r_{1}-r_{2}-\cdots-r_{i-1}(i=2, \ldots, m)
\end{array} .\right.
$$

On the curves $V_{i}$, we take $m$ Hermitian schemes as follows. For $i=1, \ldots, m$,

(A1) $S_{i}=\left\{\left(a_{1}^{i}, s_{1}^{i}\right), \ldots,\left(a_{k_{i}}^{i}, s_{k_{i}}^{i}\right)\right\}$ is a Hermitian scheme for $\mathbb{P}^{s_{i}}\left(V_{i}\right)$;

(A2) the points of $S_{i}$ do not lie on $V_{j}$ for $j<i$.

In theses conditions, the following statements hold true.

(C1) If

$$
r_{1}+r_{2}+\cdots+r_{m-1}<d<r_{1}+r_{2}+\cdots+r_{m-1}+r_{m}
$$

(C2) If

then $\left\{S_{i}, i=1, \ldots, m\right\}$ is collectable of degree $d$.

$$
r_{1}+r_{2}+\cdots+r_{m-1}<d=r_{1}+r_{2}+\cdots+r_{m-1}+r_{m}
$$

then $\left\{S_{i}, i=1, \ldots, m\right\} \cup\left\{S_{0}\right\}$ is collectable of degree $d$ where $S_{0}=\{(a, 0)\}$ and $a$ is any point not lying on the union of the $V_{i}, i=1, \ldots, m$.

In (6.6) we may consider $S_{0}=\{(a, 0)\}$ as a Hermitian scheme of degree 0 for any curve $V_{0}$ distinct from the other $V_{i}$ 's.

\begin{tabular}{|c|c|c|}
\hline Degree & Curves & Schemes on curves \\
\hline $2 m-1$ & $V_{i}$ is a quadric $(1 \leq i \leq m)$ & $\begin{array}{l}S_{i} \text { is a Hermitian scheme of order } \\
2(m-i)+1 \text { on } V_{i}\end{array}$ \\
\hline $3 m-1$ & $V_{i}$ is a cubic $(1 \leq i \leq m)$ & $\begin{array}{l}S_{i} \text { is a Hermitian scheme of order } \\
3(m-i)+2 \text { on } V_{i} \text {, for example, } \\
S_{i}=\left\{\left(a_{i}, 1\right), 1 \leq i \leq 3(m-i)-1\right\}\end{array}$ \\
\hline $\begin{array}{l}d=(d- \\
r)+r\end{array}$ & $\begin{array}{l}V_{1} \text { is a curve of degree } d- \\
r \text { and } V_{2} \text { a curve of degree } \\
r+1\end{array}$ & $\begin{array}{l}S_{1} \text { is a Hermitian scheme of degree } d \\
\text { on } V_{1} \text { and } S_{2} \text { is Hermitian scheme of } \\
\text { degree } r \text { on } V_{2} \text {. }\end{array}$ \\
\hline
\end{tabular}

Example 6.2. We give a few examples of possible configurations. Observe that to any additive partition of $d$ corresponds a configuration of type (6.6).

\subsection{Proof of Theorem 6.1}

The proof of Theorem 6.1 is an adaptation of that of [4, Theorem 4]. It is based on a simple division argument. 
We first compute the number $N$ of conditions corresponding to the family $\left\{S_{i}, i=1, \ldots, m\right\}$. Since $\mathbf{H}_{S_{i}}(f-p)=0$ gives $N_{s_{i}}\left(V_{i}\right)$ conditions, using $s_{i}-r_{i}=s_{i+1}$ for $i \leq m-1$, we have

$$
\begin{aligned}
& N=\sum_{i=1}^{m} N_{s_{i}}\left(V_{i}\right)=\sum_{i=1}^{m}\left(\begin{array}{c}
s_{i}+2 \\
2
\end{array}\right)-\left(\begin{array}{c}
s_{i}-r_{i}+2 \\
2
\end{array}\right) \quad(\text { by }(2.2)) \\
& =\left(\sum_{i=1}^{m-1}\left(\begin{array}{c}
s_{i}+2 \\
2
\end{array}\right)-\left(\begin{array}{c}
s_{i+1}+2 \\
2
\end{array}\right)\right)+\left(\begin{array}{c}
s_{m}+2 \\
2
\end{array}\right)-\left(\begin{array}{c}
s_{m}-r_{m} \\
2
\end{array}\right) \\
& =\left(\begin{array}{c}
s_{1}+2 \\
2
\end{array}\right)-\left(\begin{array}{c}
s_{m}-r_{m}+2 \\
2
\end{array}\right) \\
& = \begin{cases}\left(\begin{array}{c}
d+2 \\
2
\end{array}\right)-0=\left(\begin{array}{c}
d+2 \\
2
\end{array}\right) & \text { if (6.5) holds true } \\
\left(\begin{array}{c}
d+2 \\
2
\end{array}\right)-\left(\begin{array}{l}
2 \\
2
\end{array}\right)=\left(\begin{array}{c}
d+2 \\
2
\end{array}\right)-1 & \text { if (6.6) holds true }\end{cases}
\end{aligned}
$$

Thus, in both cases, the number of conditions equals the dimension of $\mathbb{P}^{d}\left(\mathbb{C}^{2}\right)$. To establish the theorem, it is therefore sufficient to show that if $p \in \mathbb{P}^{d}\left(\mathbb{C}^{2}\right)$ is such $\mathbf{H}_{S_{i}}(p)=0$ for every $i-$ and also, in the case (6.6), such that $p(a)=0-$ then $p$ must be the zero polynomial.

Let $p$ be such a polynomial. Since $p$ is of degree at most $s_{1}(=d)$ and $\mathbf{H}_{S_{1}}(p)=0$, we must have $p_{\mid V_{1}}=0$. Since $q_{1}$ is irreducible this implies by the Nullstellensatz that $q_{1}$ divides $p$, thus, $p=q_{1} h_{1}$ with $\operatorname{deg} h_{1}=\operatorname{deg} p-\operatorname{deg} q_{1} \leq$ $s_{1}-r_{1}=s_{2}$ that is, $h_{1} \in \mathbb{P}^{s_{2}}\left(\mathbb{C}^{2}\right)$. Now the second condition $\mathbf{H}_{S_{2}}(p)=0$ translates into $\mathbf{H}_{S_{2}}\left(q_{1} h_{1}\right)=0$. Since no point of $S_{2}$ lies on $V_{1}$ - this is assumption (6.1)$q_{1}\left(a_{j}^{2}\right) \neq 0$, for $j=1, \ldots, k_{i}^{2}$ and, in view of Lemma 5.2, $\mathbf{H}_{S_{2}}\left(h_{1}\right)=0$. Since $h_{1} \in \mathbb{P}^{s_{2}}\left(\mathbb{C}^{2}\right)$ and $S_{2}$ is Hermitian for $\mathbb{P}^{s_{2}}\left(V_{2}\right)$, we must have $h_{1} \mid V_{2}=0$ hence, again by the Nullstellensatz $h_{1}=h_{2} q_{2}$ with $h_{2} \in \mathbb{P}^{s_{3}}\left(\mathbb{C}^{2}\right)$. The third condition now translates into $\mathbf{H}_{S_{3}}\left(h_{2} q_{1} q_{2}\right)=0$. Again, since $q_{1} q_{2} \neq 0$ vanishes on no point of $S_{3}$, Lemma 5.2 yields $\mathbf{H}_{S_{3}}\left(h_{2}\right)=0$ and since $\operatorname{deg} h_{2} \leq s_{3}$ and $S_{3}$ is Hermitian for $\mathbb{P}^{s_{3}}\left(V_{3}\right)$ we must have $h_{2}=0$ on $V_{3}$, hence $h_{2}=h_{3} q_{3}$ Continuing in this way we arrive at $p=q_{1} q_{2} \ldots q_{m} h_{m}$ with some polynomial $h_{m}$. When (6.5) holds, comparing the degree of both sides, we deduce at once that $h_{m}$ must be zero which gives in turn $p=0$, whereas when (6.6) holds, $h_{m}$ must be a constant polynomial and the use of the condition $p(a)=0$ forces this constant to be zero (for no $q_{i}(a)$ vanishes) which again permits us to conclude that $p=0$ and this finishes the proof.

\section{References}

[1] B. BoJAnOv and Y. XU., On polynomial interpolation of two variables, J. Approx. Theory 120 (2003), 267-282. 
[2] B. D. Bojanov, H. A. Hakopian and A. A. Sahakian, "Spline Functions and Multivariate Interpolations", Mathematics and its Applications, Vol. 248, Academic Publishers Group, Dordrecht, 1993.

[3] L. Bos, On certain configurations of points in $\mathbf{R}^{n}$ which are unisolvent for polynomial interpolation, J. Approx. Theory 64 (1991), 271-280.

[4] L. P. Bos and J.-P. CALVI, Multipoint taylor interpolation, Calcolo 51 (2008), 35-51.

[5] J.-P. CALVI and L. FILIPSSON, The polynomial projectors that preserve homogeneous differential relations: a new characterization of Kergin interpolation, East J. Approx. 10 (2004), 441-454.

[6] D. Cox, J. LitTle and D. O'SheA, “Ideals, Varieties, and Algorithms", Undergraduate Texts in Mathematics, Springer, New York, third edition, 2007.

[7] C. DE BOOR and A. RON, On multivariate polynomial interpolation, Constr. Approx. 6 (1990), 287-302.

[8] C. DE BOOR and A. RON, The least solution for the polynomial interpolation problem, Math. Z. 210 (1992), 347-378.

[9] LARS FILIPSSON, Complex mean-value interpolation and approximation of holomorphic functions, J. Approx. Theory 91 (1997), 244-278.

[10] M. GASCA and T. SAUER, Polynomial interpolation in several variables, Adv. Comput. Math. 12 (2000), 377-410. Multivariate polynomial interpolation.

[11] H. A. HAKOPIAN and M. F. KHALAF, On the poisedness of Bojanov-Xu interpolation, J. Approx. Theory 135 (2005), 176-202.

[12] H. A. HAKOPIAN and M. F. KHALAF, On the poisedness of Bojanov-Xu interpolation, II, East J. Approx. 11 (2005), 187-220.

[13] F. KIRWAN, "Complex Algebraic Curves", London Mathematical Society Student Texts, Vol. 23, Cambridge University Press, Cambridge, 1992.

[14] R. A. LoREnTz, "Multivariate Birkhoff Interpolation", Lecture Notes in Mathematics, Vol. 1516. ix, Springer-Verlag, 1992.

[15] R. A. Lorentz, Multivariate Hermite interpolation by algebraic polynomials: A survey, J. Comput. Appl. Math. 122 (2000), 167-201.

[16] M. G. MARINARI, H. M. MöLler and T. Mora, Gröbner bases of ideals defined by functionals with an application to ideals of projective points, Appl. Algebra Engrg. Comm. Comput. 4 (1993), 103-145.

[17] H. M. MöLLER, Hermite interpolation in several variables using ideal-theoretic methods, In: "Constructive Theory of Functions of Several Variables", Proc. Conf., Math. Res. Inst., Oberwolfach, 1976, Lecture Notes in Math., Vol. 571, Springer, Berlin, 1977, 155-163.

Department of Mathematics

University of Calgary

Calgary, Alberta, T2N1N4 Canada

lpbos@math.ucalgary.ca

Institut de Mathématiques

Université de Toulouse III

and CNRS (UMR 5219)

31062, Toulouse Cedex 9, France

jean-paul.calvi@math.ups-tlse.fr 